\title{
Dinitrogen Complexes of Pincer-Ligated Iridium
}

\author{
Rajshekhar Ghosh, Mira Kanzelberger, Thomas J. Emge, \\ Gene S. Hall and Alan S. Goldman* \\ Department of Chemistry and Chemical Biology, \\ Rutgers-The State University of New Jersey, Piscataway, New Jersey, 08854
}

\section{Supporting Information}

Page \#

I. Experimental section: Raman and Infrared measurements S2

II. X-ray Structure Determinations $\quad$ S3

III. Structural Data for complex 1

(a) Figure S-1. ORTEP Diagram of complex $1 \quad$ S4

(b) Table S-1. Crystal data and structure refinement for complex $1 \quad$ S5

(c) Table S-2. Atomic coordinates for complex $1 \quad$ S6

(d) Table S-3. Selected bond lengths and angles for complex $1 \quad$ S8

(e) Table S-4. Torsion angles for complex $1 \quad$ S11

IV. Structural Data for complex 2

$\begin{array}{ll}\text { (a) Figure S-2. ORTEP Diagram of complex } 2 & \text { S14 }\end{array}$

(b) Table S-5. Crystal data and structure refinement for complex $2 \quad \mathrm{~S} 15$

(c) Table S-6. Atomic coordinates for complex $2 \quad$ S16

(d) Table S-7. Selected bond lengths and angles for complex $2 \quad$ S17

(e) Table S-8. Torsion angles for complex $2 \quad$ S21 


\section{Experimental Section}

Raman Spectra of complexes 1 and 2: Room temperature $\left(21 \pm 1^{\circ} \mathrm{C}\right)$ Raman spectra were obtained from a Renishaw (Renishaw Inc., Hoffman Estates, IL) System 1000 dispersive micro-Raman spectrometer. The system has a $785 \mathrm{~nm}$ diode laser for illuminating the sample and a holographic notch filter to minimize the Raleigh scattered laser line. The Raman photons were focused onto a 1200 1/mm holographic grating and sent to a Peltier CCD detector for analysis. The laser intensity at the sample was $25 \mathrm{~mW}$ and we used a 50x objective lens from a Leica microscope to project the laser ( 2 micron spot) onto the sample. The laser beam was focused onto the sample crystals thru a sealed-glass NMR tube. We used acetaminophen (ASTM Designation: E 1840 - 96) to calibrate the Raman spectrometer over the data collection range of 2150 to $1860 \mathrm{~cm}^{-1}$ at 2 $\mathrm{cm}^{-1}$ resolution. The micro-Raman spectrometer is equipped with a computer controlled $\mathrm{x}, \mathrm{y}, \mathrm{z}$ stage. Under the control of Renishaw Wire 2.0 data acquisition software, data collection consisted of 3, 20 second accumulations per spot that were co-added to form an averaged data point per sample. Three separate random locations in the NMR tube were sampled to determine sample homogeneity that was confirmed by analysis of the three Raman spectra.

Infrared Spectra for complex 1: Room temperature $\left(21 \pm 1^{\circ} \mathrm{C}\right)$ attenuated total reflectance (ATR) FTIR spectra were recorded on a Bruker (Billerica, MA) Equinox55 spectrometer equipped with a DTGS detector and KBr beamsplitter. We used a Smiths Detection (Danbury, CT) single reflection DuraScope ATR attachment that had a diamond crystal and a KRS-5 focusing element. Spectra were collected by pressing the sample onto the 2-mm diameter diamond crystal with a pressure setting of 6 on the DuraScope. One-hundred co-added spectra were accumulated between 4,000 and 400 $\mathrm{cm}^{-1}$ at $4 \mathrm{~cm}^{-1}$ resolution using the Blackman-Harris 3-term apodization function. All spectra were normalized by subtracting out a background spectra collected before each sample analysis. Spectra were analyzed as is with no further spectral manipulations. 
II. X-ray Structure Determinations. Data were collected on a Bruker Smart APEX CCD diffractometer with graphite monochromatized Mo K $\alpha$ radiation $(\lambda=$ $0.71073 \AA$ ) at $100 \mathrm{~K}$. The data were corrected for Lorenz effects and polarization, and absorption, the latter by a multi-scan (SADABS) ${ }^{1}$ method. The structures were solved by Patterson or direct methods (SHELXS86). ${ }^{2}$ All non-hydrogen atoms were refined $(\mathrm{SHELXL} 97)^{3}$ based upon $\mathrm{F}_{\mathrm{obs}}{ }^{2}$. All hydrogen atom coordinates were calculated with idealized geometries (SHELXL97). Scattering factors $\left(f_{o}, f^{\prime}, f^{\prime \prime}\right)$ are as described in SHELXL97. Crystal data for the structures have been deposited in the Cambridge Crystallogaphic Data Center with numbers (compound numbers) CCDC- 616700 (complex 1), CCDC- 616699 (complex 2).

\section{References}

(1) Bruker-AXS. SADABS, Bruker area detector scaling and absorption correction, v2.05, Bruker-AXS Inc., Madison, Wisconsin, 2003; SAINTplus, Bruker area detector data reduction program, v6.45, Bruker-AXS Inc., Madison, Wisconsin, 2003.

(2) Sheldrick, G. M. SHELXS86, Program for the Solution of Crystal Structures, University of Göttingen, Germany, 1986.

(3) Sheldrick, G. M. SHELXL97, Program for Crystal Structure Refinement, University of Göttingen, Germany, 1997. 
III. Structural Data for 1

Figure S-1 : ORTEP Diagram of complex 1
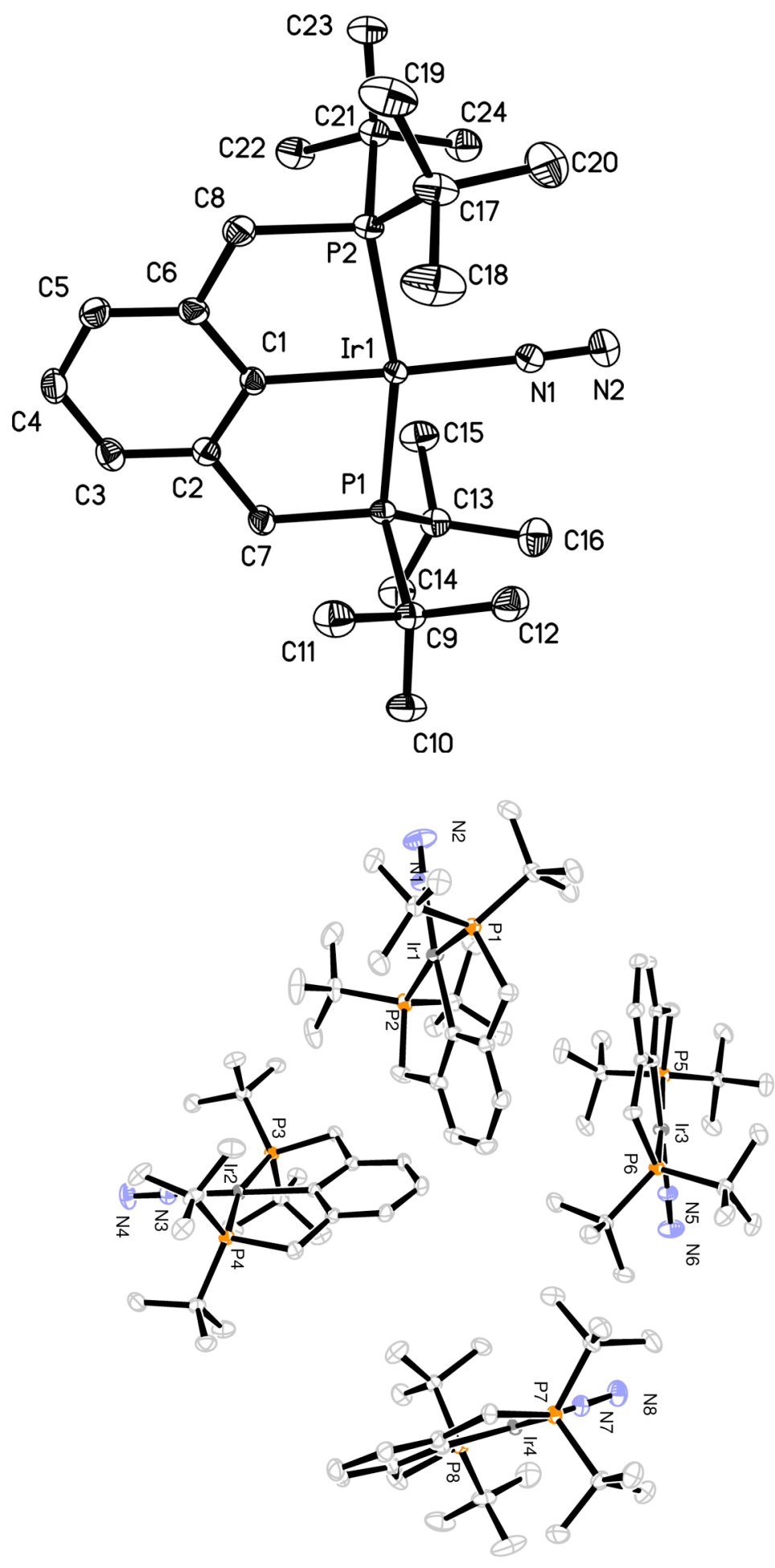
Table S-1. Crystal data and structure refinement for complex 1 .

Identification code

Empirical formula

Formula weight

Temperature

Wavelength

Crystal system

Space group

Unit cell dimensions

Volume

Z

Density (calculated)

Absorption coefficient

$\mathrm{F}(000)$

Crystal size

Theta range for data collection

Index ranges

Reflections collected

Independent reflections

Completeness to theta $=30.85^{\circ}$

Absorption correction

Max. and min. transmission

Refinement method

Data / restraints / parameters

Goodness-of-fit on $\mathrm{F}^{2}$

Final $R$ indices [I $>2 \operatorname{sigma}(\mathrm{I})]$

$\mathrm{R}$ indices (all data)

Largest diff. peak and hole $\operatorname{irn} 2 \mathrm{zzZ}$

C24 H43 Ir N2 P2

613.74

100(2) K

$0.71073 \AA$

Triclinic

P-1

$$
\begin{array}{ll}
\mathrm{a}=11.2546(8) \AA & \alpha=89.034(1)^{\circ} . \\
\mathrm{b}=15.8510(11) \AA & \beta=81.718(1)^{\circ} . \\
\mathrm{c}=29.533(2) \AA & \gamma=88.825(1)^{\circ} .
\end{array}
$$

$5212.0(6) \AA^{3}$

8

$1.564 \mathrm{Mg} / \mathrm{m}^{3}$

$5.259 \mathrm{~mm}^{-1}$

2464

$0.30 \times 0.11 \times 0.10 \mathrm{~mm}^{3}$

1.83 to $30.85^{\circ}$.

$-16<=\mathrm{h}<=16,-22<=\mathrm{k}<=22,-42<=1<=42$

62903

$31856[\mathrm{R}(\mathrm{int})=0.0158]$

$97.2 \%$

Semi-empirical from equivalents

0.9999 and 0.8068

Full-matrix least-squares on $\mathrm{F}^{2}$

$31856 / 0 / 1093$

1.011

$\mathrm{R} 1=0.0202, \mathrm{wR} 2=0.0421$

$\mathrm{R} 1=0.0235, \mathrm{wR} 2=0.0431$

1.260 and -0.705 e. $\AA^{-3}$ 
Table S-2. Atomic coordinates ( $\left.\times 10^{4}\right)$ and equivalent isotropic displacement parameters $\left(\AA^{2} \times 10^{3}\right)$ for complex 1. $U(\mathrm{eq})$ is defined as one third of the trace of the orthogonalized $\mathrm{U}^{\mathrm{ij}}$ tensor.

\begin{tabular}{|c|c|c|c|c|}
\hline & $\mathrm{x}$ & $\mathrm{y}$ & $\mathrm{z}$ & $\mathrm{U}(\mathrm{eq})$ \\
\hline$\overline{\operatorname{Ir}}(1)$ & $5295(1)$ & $1178(1)$ & $6128(1)$ & $14(1)$ \\
\hline $\mathrm{N}(1)$ & $4740(2)$ & $367(1)$ & $5719(1)$ & $25(1)$ \\
\hline $\mathrm{N}(2)$ & $4396(2)$ & $-118(2)$ & $5509(1)$ & $40(1)$ \\
\hline $\mathrm{P}(1)$ & $7161(1)$ & $590(1)$ & $6143(1)$ & $15(1)$ \\
\hline $\mathrm{P}(2)$ & $3575(1)$ & $1990(1)$ & $6240(1)$ & $15(1)$ \\
\hline $\mathrm{C}(1)$ & $5814(2)$ & $1970(1)$ & $6597(1)$ & $15(1)$ \\
\hline $\mathrm{C}(2)$ & $6882(2)$ & $1827(1)$ & $6794(1)$ & $17(1)$ \\
\hline $\mathrm{C}(3)$ & $7201(2)$ & $2348(1)$ & $7131(1)$ & 21(1) \\
\hline C(4) & $6454(2)$ & $3011(1)$ & $7299(1)$ & $23(1)$ \\
\hline $\mathrm{C}(5)$ & $5397(2)$ & $3167(1)$ & $7119(1)$ & $22(1)$ \\
\hline$C(6)$ & $5095(2)$ & $2675(1)$ & $6768(1)$ & $18(1)$ \\
\hline $\mathrm{C}(7)$ & $7668(2)$ & $1060(1)$ & $6649(1)$ & $19(1)$ \\
\hline $\mathrm{C}(8)$ & $3991(2)$ & $2905(1)$ & $6551(1)$ & $22(1)$ \\
\hline$C(9)$ & $8289(2)$ & 911(1) & $5642(1)$ & $19(1)$ \\
\hline$C(10)$ & $9538(2)$ & $496(2)$ & $5627(1)$ & $27(1)$ \\
\hline$C(11)$ & $8407(2)$ & $1876(1)$ & $5670(1)$ & $28(1)$ \\
\hline$C(12)$ & $7789(2)$ & $727(2)$ & $5196(1)$ & $26(1)$ \\
\hline$C(13)$ & $7272(2)$ & $-577(1)$ & $6267(1)$ & $20(1)$ \\
\hline$C(14)$ & $8425(2)$ & $-860(1)$ & $6452(1)$ & $26(1)$ \\
\hline$C(15)$ & $6198(2)$ & $-766(1)$ & $6636(1)$ & $28(1)$ \\
\hline$C(16)$ & $7152(2)$ & $-1090(1)$ & $5839(1)$ & $29(1)$ \\
\hline$C(17)$ & $3006(2)$ & $2482(2)$ & $5727(1)$ & $25(1)$ \\
\hline $\mathrm{C}(18)$ & $4128(2)$ & $2811(2)$ & $5420(1)$ & $43(1)$ \\
\hline$C(19)$ & $2124(2)$ & $3229(2)$ & $5837(1)$ & $35(1)$ \\
\hline $\mathrm{C}(20)$ & $2417(2)$ & $1820(2)$ & $5463(1)$ & $38(1)$ \\
\hline $\mathrm{C}(21)$ & $2340(2)$ & $1472(1)$ & $6638(1)$ & $17(1)$ \\
\hline $\mathrm{C}(22)$ & $2779(2)$ & $1413(2)$ & $7108(1)$ & $26(1)$ \\
\hline $\mathrm{C}(23)$ & $1129(2)$ & $1943(1)$ & $6685(1)$ & $24(1)$ \\
\hline$C(24)$ & $2192(2)$ & $570(1)$ & $6477(1)$ & $26(1)$ \\
\hline $\operatorname{Ir}(2)$ & $332(1)$ & $6039(1)$ & $6207(1)$ & $13(1)$ \\
\hline $\mathrm{N}(3)$ & $-130(2)$ & $6793(1)$ & $5734(1)$ & 21(1) \\
\hline $\mathrm{N}(4)$ & $-400(2)$ & $7228(1)$ & $5468(1)$ & $31(1)$ \\
\hline $\mathrm{P}(3)$ & $-1379(1)$ & $5255(1)$ & $6316(1)$ & $13(1)$ \\
\hline $\mathrm{P}(4)$ & $2128(1)$ & $6644(1)$ & $6268(1)$ & $14(1)$ \\
\hline$C(25)$ & $815(2)$ & $5241(1)$ & $6704(1)$ & $14(1)$ \\
\hline$C(26)$ & $110(2)$ & $4537(1)$ & $6872(1)$ & $16(1)$ \\
\hline $\mathrm{C}(27)$ & $430(2)$ & $4003(1)$ & $7215(1)$ & $19(1)$ \\
\hline $\mathrm{C}(28)$ & $1482(2)$ & $4126(1)$ & $7395(1)$ & $22(1)$ \\
\hline$C(29)$ & $2202(2)$ & 4801(1) & $7240(1)$ & $20(1)$ \\
\hline$C(30)$ & $1870(2)$ & $5361(1)$ & $6907(1)$ & $16(1)$ \\
\hline$C(31)$ & $-992(2)$ & $4339(1)$ & $6657(1)$ & $18(1)$ \\
\hline$C(32)$ & $2613(2)$ & $6132(1)$ & $6777(1)$ & $19(1)$ \\
\hline$C(33)$ & $-1906(2)$ & $4754(1)$ & $5810(1)$ & $18(1)$ \\
\hline$C(34)$ & $-2816(2)$ & $4046(1)$ & $5935(1)$ & $23(1)$ \\
\hline$C(35)$ & $-765(2)$ & $4373(1)$ & $5530(1)$ & $25(1)$ \\
\hline$C(36)$ & $-2440(2)$ & $5417(1)$ & $5510(1)$ & $26(1)$ \\
\hline$C(37)$ & $-2627(2)$ & $5815(1)$ & $6690(1)$ & $17(1)$ \\
\hline $\mathrm{C}(38)$ & $-3839(2)$ & $5373(1)$ & $6748(1)$ & $22(1)$ \\
\hline C(39) & $-2216(2)$ & $5892(2)$ & $7164(1)$ & $26(1)$ \\
\hline$C(40)$ & $-2768(2)$ & $6713(1)$ & $6498(1)$ & $24(1)$ \\
\hline$C(41)$ & $3356(2)$ & $6393(1)$ & $5789(1)$ & 21(1) \\
\hline$C(42)$ & $3526(2)$ & $5426(2)$ & $5799(1)$ & $33(1)$ \\
\hline$C(43)$ & $4555(2)$ & $6810(2)$ & $5823(1)$ & $25(1)$ \\
\hline$C(44)$ & 2947(2) & $6636(2)$ & $5330(1)$ & $29(1)$ \\
\hline$C(45)$ & $2100(2)$ & $7799(1)$ & $6410(1)$ & $18(1)$ \\
\hline$C(46)$ & 999(2) & $7924(1)$ & $6775(1)$ & $26(1)$ \\
\hline$C(47)$ & $3208(2)$ & $8102(1)$ & $6605(1)$ & $24(1)$ \\
\hline $\mathrm{C}(48)$ & $1927(2)$ & $8344(1)$ & $5989(1)$ & $27(1)$ \\
\hline $\operatorname{Ir}(3)$ & $1495(1)$ & $1051(1)$ & $8785(1)$ & $13(1)$ \\
\hline
\end{tabular}




\begin{tabular}{|c|c|c|c|c|}
\hline $\mathrm{N}(5)$ & $604(2)$ & $1782(1)$ & $9243(1)$ & $20(1)$ \\
\hline $\mathrm{N}(6)$ & $95(2)$ & $2200(1)$ & $9501(1)$ & $30(1)$ \\
\hline $\mathrm{P}(5)$ & $-85(1)$ & 248(1) & $8647(1)$ & $14(1)$ \\
\hline $\mathrm{P}(6)$ & $3341(1)$ & $1639(1)$ & $8783(1)$ & $12(1)$ \\
\hline C(49) & $2432(2)$ & 298(1) & $8297(1)$ & $13(1)$ \\
\hline $\mathrm{C}(50)$ & $1901(2)$ & $-397(1)$ & $8112(1)$ & $14(1)$ \\
\hline$C(51)$ & $2533(2)$ & $-886(1)$ & $7765(1)$ & $16(1)$ \\
\hline$C(52)$ & $3727(2)$ & $-728(1)$ & $7604(1)$ & $18(1)$ \\
\hline $\mathrm{C}(53)$ & $4290(2)$ & $-64(1)$ & $7783(1)$ & $17(1)$ \\
\hline$C(54)$ & $3649(2)$ & $449(1)$ & $8116(1)$ & $15(1)$ \\
\hline$C(55)$ & $630(2)$ & $-632(1)$ & $8307(1)$ & $17(1)$ \\
\hline$C(56)$ & $4256(2)$ & $1214(1)$ & $8269(1)$ & $16(1)$ \\
\hline$C(57)$ & $-1064(2)$ & 802(1) & $8265(1)$ & $18(1)$ \\
\hline $\mathrm{C}(58)$ & $-2180(2)$ & $326(1)$ & $8179(1)$ & $25(1)$ \\
\hline$C(59)$ & $-260(2)$ & $950(2)$ & $7806(1)$ & $29(1)$ \\
\hline$C(60)$ & $-1443(2)$ & $1671(1)$ & $8463(1)$ & $24(1)$ \\
\hline $\mathrm{C}(61)$ & $-1008(2)$ & $-295(1)$ & $9145(1)$ & $18(1)$ \\
\hline$C(62)$ & $-1769(2)$ & $-1022(1)$ & $9011(1)$ & $26(1)$ \\
\hline$C(63)$ & $-101(2)$ & $-657(2)$ & $9444(1)$ & $26(1)$ \\
\hline$C(64)$ & $-1824(2)$ & $348(1)$ & $9429(1)$ & $26(1)$ \\
\hline$C(65)$ & $3479(2)$ & $2811(1)$ & $8696(1)$ & $16(1)$ \\
\hline$C(66)$ & $2715(2)$ & $3034(1)$ & $8317(1)$ & $24(1)$ \\
\hline$C(67)$ & $4764(2)$ & $3113(1)$ & $8534(1)$ & $22(1)$ \\
\hline $\mathrm{C}(68)$ & $2954(2)$ & $3284(1)$ & $9129(1)$ & $23(1)$ \\
\hline C(69) & $4121(2)$ & $1260(1)$ & $9271(1)$ & $18(1)$ \\
\hline$C(70)$ & $4267(2)$ & 295(1) & $9221(1)$ & $28(1)$ \\
\hline $\mathrm{C}(71)$ & $5352(2)$ & $1643(1)$ & $9279(1)$ & $23(1)$ \\
\hline$C(72)$ & $3313(2)$ & $1431(1)$ & $9728(1)$ & $23(1)$ \\
\hline $\operatorname{Ir}(4)$ & $6240(1)$ & $6078(1)$ & $8828(1)$ & $13(1)$ \\
\hline$N(7)$ & $5373(2)$ & $5264(1)$ & $9246(1)$ & $19(1)$ \\
\hline $\mathrm{N}(8)$ & $4881(2)$ & $4790(1)$ & $9479(1)$ & $29(1)$ \\
\hline $\mathrm{P}(7)$ & $8123(1)$ & $5510(1)$ & $8840(1)$ & $14(1)$ \\
\hline $\mathrm{P}(8)$ & $4621(1)$ & $6906(1)$ & $8708(1)$ & $15(1)$ \\
\hline$C(73)$ & $7150(2)$ & $6906(1)$ & $8372(1)$ & $15(1)$ \\
\hline$C(74)$ & $8385(2)$ & $6789(1)$ & $8202(1)$ & $16(1)$ \\
\hline$C(75)$ & $8990(2)$ & $7344(1)$ & $7880(1)$ & $20(1)$ \\
\hline$C(76)$ & $8389(2)$ & $8018(1)$ & $7703(1)$ & $24(1)$ \\
\hline$C(77)$ & $7177(2)$ & $8151(1)$ & $7863(1)$ & $23(1)$ \\
\hline$C(78)$ & $6574(2)$ & $7622(1)$ & $8198(1)$ & $18(1)$ \\
\hline$C(79)$ & $9045(2)$ & $6017(1)$ & $8349(1)$ & $17(1)$ \\
\hline $\mathrm{C}(80)$ & $5290(2)$ & $7833(1)$ & $8396(1)$ & $24(1)$ \\
\hline $\mathrm{C}(81)$ & $8383(2)$ & $4352(1)$ & $8724(1)$ & $18(1)$ \\
\hline $\mathrm{C}(82)$ & $9705(2)$ & 4092(1) & $8578(1)$ & $23(1)$ \\
\hline $\mathrm{C}(83)$ & $7683(2)$ & $4170(1)$ & $8328(1)$ & $25(1)$ \\
\hline $\mathrm{C}(84)$ & $7865(2)$ & $3814(1)$ & $9143(1)$ & $26(1)$ \\
\hline$C(85)$ & $8790(2)$ & $5834(1)$ & $9358(1)$ & $18(1)$ \\
\hline$C(86)$ & $10035(2)$ & $5446(2)$ & $9393(1)$ & $25(1)$ \\
\hline $\mathrm{C}(87)$ & $8889(2)$ & $6800(1)$ & $9328(1)$ & $27(1)$ \\
\hline $\mathrm{C}(88)$ & $7921(2)$ & $5615(2)$ & $9792(1)$ & $24(1)$ \\
\hline C(89) & $3662(2)$ & $6428(1)$ & $8313(1)$ & $18(1)$ \\
\hline $\mathrm{C}(90)$ & $4468(2)$ & $6346(2)$ & $7849(1)$ & $30(1)$ \\
\hline $\mathrm{C}(91)$ & $2523(2)$ & $6934(1)$ & $8244(1)$ & $27(1)$ \\
\hline$C(92)$ & $3315(2)$ & $5535(1)$ & $8484(1)$ & $24(1)$ \\
\hline$C(93)$ & $3682(2)$ & $7380(1)$ & $9221(1)$ & $26(1)$ \\
\hline $\mathrm{C}(94)$ & $2903(2)$ & $8142(2)$ & $9113(1)$ & $37(1)$ \\
\hline$C(95)$ & $2872(2)$ & $6719(2)$ & $9487(1)$ & $38(1)$ \\
\hline$C(96)$ & $4589(2)$ & $7669(2)$ & $9528(1)$ & $47(1)$ \\
\hline
\end{tabular}


Table S-3. Bond lengths $[\AA]$ and angles $\left[^{\circ}\right]$ for complex $\mathbf{1}$.

\begin{tabular}{|c|c|c|c|}
\hline $\operatorname{Ir}(1)-\mathrm{N}(1)$ & $1.9489(18)$ & $\operatorname{Ir}(3)-\mathrm{N}(5)$ & $1.9472(17)$ \\
\hline $\operatorname{Ir}(1)-C(1)$ & $2.0398(19)$ & $\operatorname{Ir}(3)-C(49)$ & $2.0452(18)$ \\
\hline $\operatorname{Ir}(1)-P(1)$ & $2.2867(5)$ & $\operatorname{Ir}(3)-P(6)$ & $2.2936(5)$ \\
\hline $\operatorname{Ir}(1)-P(2)$ & $2.2897(5)$ & $\operatorname{Ir}(3)-P(5)$ & $2.2945(5)$ \\
\hline $\mathrm{N}(1)-\mathrm{N}(2)$ & $1.107(3)$ & $\mathrm{N}(5)-\mathrm{N}(6)$ & $1.107(2)$ \\
\hline $\mathrm{P}(1)-\mathrm{C}(7)$ & $1.846(2)$ & $\mathrm{P}(5)-\mathrm{C}(55)$ & $1.8367(19)$ \\
\hline $\mathrm{P}(1)-\mathrm{C}(9)$ & $1.877(2)$ & $\mathrm{P}(5)-\mathrm{C}(61)$ & $1.8802(19)$ \\
\hline $\mathrm{P}(1)-\mathrm{C}(13)$ & $1.883(2)$ & $P(5)-C(57)$ & $1.8825(19)$ \\
\hline $\mathrm{P}(2)-\mathrm{C}(8)$ & $1.835(2)$ & $P(6)-C(56)$ & $1.8361(19)$ \\
\hline $\mathrm{P}(2)-\mathrm{C}(21)$ & $1.8790(19)$ & $\mathrm{P}(6)-\mathrm{C}(69)$ & $1.877(2)$ \\
\hline $\mathrm{P}(2)-\mathrm{C}(17)$ & $1.879(2)$ & $P(6)-C(65)$ & $1.8777(19)$ \\
\hline $\mathrm{C}(1)-\mathrm{C}(2)$ & $1.421(3)$ & $\mathrm{C}(49)-\mathrm{C}(50)$ & $1.417(3)$ \\
\hline$C(1)-C(6)$ & $1.422(3)$ & $C(49)-C(54)$ & $1.421(2)$ \\
\hline$C(2)-C(3)$ & $1.396(3)$ & $\mathrm{C}(50)-\mathrm{C}(51)$ & $1.397(3)$ \\
\hline$C(2)-C(7)$ & $1.518(3)$ & $\mathrm{C}(50)-\mathrm{C}(55)$ & $1.514(3)$ \\
\hline$C(3)-C(4)$ & $1.386(3)$ & $\mathrm{C}(51)-\mathrm{C}(52)$ & $1.386(3)$ \\
\hline $\mathrm{C}(4)-\mathrm{C}(5)$ & $1.388(3)$ & $\mathrm{C}(52)-\mathrm{C}(53)$ & $1.389(3)$ \\
\hline$C(5)-C(6)$ & $1.391(3)$ & $\mathrm{C}(53)-\mathrm{C}(54)$ & $1.395(2)$ \\
\hline $\mathrm{C}(6)-\mathrm{C}(8)$ & $1.514(3)$ & $C(54)-C(56)$ & $1.511(3)$ \\
\hline $\mathrm{C}(9)-\mathrm{C}(10)$ & $1.535(3)$ & $\mathrm{C}(57)-\mathrm{C}(58)$ & $1.532(3)$ \\
\hline $\mathrm{C}(9)-\mathrm{C}(12)$ & $1.540(3)$ & $\mathrm{C}(57)-\mathrm{C}(59)$ & $1.534(3)$ \\
\hline C(9)-C(11) & $1.542(3)$ & $C(57)-C(60)$ & $1.535(3)$ \\
\hline$C(13)-C(14)$ & $1.536(3)$ & $\mathrm{C}(61)-\mathrm{C}(64)$ & $1.533(3)$ \\
\hline $\mathrm{C}(13)-\mathrm{C}(15)$ & $1.537(3)$ & $\mathrm{C}(61)-\mathrm{C}(63)$ & $1.538(3)$ \\
\hline $\mathrm{C}(13)-\mathrm{C}(16)$ & $1.538(3)$ & $\mathrm{C}(61)-\mathrm{C}(62)$ & $1.540(3)$ \\
\hline$C(17)-C(20)$ & $1.534(4)$ & $\mathrm{C}(65)-\mathrm{C}(68)$ & $1.532(3)$ \\
\hline $\mathrm{C}(17)-\mathrm{C}(19)$ & $1.538(3)$ & $C(65)-C(66)$ & $1.536(3)$ \\
\hline $\mathrm{C}(17)-\mathrm{C}(18)$ & $1.541(3)$ & $C(65)-C(67)$ & $1.540(3)$ \\
\hline$C(21)-C(23)$ & $1.530(3)$ & $C(69)-C(71)$ & $1.528(3)$ \\
\hline $\mathrm{C}(21)-\mathrm{C}(24)$ & $1.535(3)$ & $\mathrm{C}(69)-\mathrm{C}(72)$ & $1.538(3)$ \\
\hline $\mathrm{C}(21)-\mathrm{C}(22)$ & $1.538(3)$ & $C(69)-C(70)$ & $1.542(3)$ \\
\hline $\operatorname{Ir}(2)-\mathrm{N}(3)$ & $1.9442(17)$ & $\operatorname{Ir}(4)-N(7)$ & $1.9482(17)$ \\
\hline $\operatorname{Ir}(2)-C(25)$ & $2.0483(18)$ & $\operatorname{Ir}(4)-C(73)$ & $2.0446(18)$ \\
\hline $\operatorname{Ir}(2)-P(4)$ & $2.2867(5)$ & $\operatorname{Ir}(4)-\mathrm{P}(8)$ & $2.2894(5)$ \\
\hline $\operatorname{Ir}(2)-P(3)$ & $2.2940(5)$ & $\operatorname{Ir}(4)-P(7)$ & $2.2902(5)$ \\
\hline $\mathrm{N}(3)-\mathrm{N}(4)$ & $1.107(2)$ & $\mathrm{N}(7)-\mathrm{N}(8)$ & $1.109(2)$ \\
\hline $\mathrm{P}(3)-\mathrm{C}(31)$ & $1.8337(19)$ & $P(7)-C(79)$ & $1.8412(19)$ \\
\hline$P(3)-C(37)$ & $1.8744(19)$ & $\mathrm{P}(7)-\mathrm{C}(85)$ & $1.880(2)$ \\
\hline $\mathrm{P}(3)-\mathrm{C}(33)$ & $1.880(2)$ & $\mathrm{P}(7)-\mathrm{C}(81)$ & $1.883(2)$ \\
\hline $\mathrm{P}(4)-\mathrm{C}(32)$ & $1.8411(19)$ & $\mathrm{P}(8)-\mathrm{C}(80)$ & $1.838(2)$ \\
\hline $\mathrm{P}(4)-\mathrm{C}(41)$ & $1.872(2)$ & $\mathrm{P}(8)-\mathrm{C}(93)$ & $1.875(2)$ \\
\hline $\mathrm{P}(4)-\mathrm{C}(45)$ & $1.883(2)$ & $\mathrm{P}(8)-\mathrm{C}(89)$ & $1.878(2)$ \\
\hline$C(25)-C(30)$ & $1.421(3)$ & $C(73)-C(74)$ & $1.418(3)$ \\
\hline$C(25)-C(26)$ & $1.422(3)$ & $\mathrm{C}(73)-\mathrm{C}(78)$ & $1.421(3)$ \\
\hline$C(26)-C(27)$ & $1.392(3)$ & $\mathrm{C}(74)-\mathrm{C}(75)$ & $1.396(3)$ \\
\hline $\mathrm{C}(26)-\mathrm{C}(31)$ & $1.511(3)$ & $\mathrm{C}(74)-\mathrm{C}(79)$ & $1.508(3)$ \\
\hline $\mathrm{C}(27)-\mathrm{C}(28)$ & $1.385(3)$ & $C(75)-C(76)$ & $1.388(3)$ \\
\hline $\mathrm{C}(28)-\mathrm{C}(29)$ & $1.386(3)$ & $C(76)-C(77)$ & $1.390(3)$ \\
\hline$C(29)-C(30)$ & $1.399(3)$ & $\mathrm{C}(77)-\mathrm{C}(78)$ & $1.394(3)$ \\
\hline$C(30)-C(32)$ & $1.509(3)$ & $\mathrm{C}(78)-\mathrm{C}(80)$ & $1.512(3)$ \\
\hline $\mathrm{C}(33)-\mathrm{C}(36)$ & $1.531(3)$ & $\mathrm{C}(81)-\mathrm{C}(83)$ & $1.537(3)$ \\
\hline$C(33)-C(34)$ & $1.540(3)$ & $\mathrm{C}(81)-\mathrm{C}(82)$ & $1.538(3)$ \\
\hline$C(33)-C(35)$ & $1.541(3)$ & $\mathrm{C}(81)-\mathrm{C}(84)$ & $1.540(3)$ \\
\hline $\mathrm{C}(37)-\mathrm{C}(38)$ & $1.533(3)$ & $\mathrm{C}(85)-\mathrm{C}(86)$ & $1.535(3)$ \\
\hline $\mathrm{C}(37)-\mathrm{C}(40)$ & $1.536(3)$ & $\mathrm{C}(85)-\mathrm{C}(88)$ & $1.536(3)$ \\
\hline $\mathrm{C}(37)-\mathrm{C}(39)$ & $1.545(3)$ & $\mathrm{C}(85)-\mathrm{C}(87)$ & $1.538(3)$ \\
\hline$C(41)-C(43)$ & $1.532(3)$ & $\mathrm{C}(89)-\mathrm{C}(92)$ & $1.534(3)$ \\
\hline $\mathrm{C}(41)-\mathrm{C}(44)$ & $1.534(3)$ & $\mathrm{C}(89)-\mathrm{C}(91)$ & $1.535(3)$ \\
\hline$C(41)-C(42)$ & $1.542(3)$ & $\mathrm{C}(89)-\mathrm{C}(90)$ & $1.536(3)$ \\
\hline$C(45)-C(46)$ & $1.532(3)$ & $\mathrm{C}(93)-\mathrm{C}(95)$ & $1.534(4)$ \\
\hline$C(45)-C(47)$ & $1.535(3)$ & $\mathrm{C}(93)-\mathrm{C}(94)$ & $1.534(3)$ \\
\hline$C(45)-C(48)$ & $1.537(3)$ & $\mathrm{C}(93)-\mathrm{C}(96)$ & $1.542(3)$ \\
\hline
\end{tabular}




\begin{tabular}{|c|c|}
\hline$N(1)-\operatorname{Ir}(1)-C(1)$ & $175.51(8)$ \\
\hline$N(1)-\operatorname{Ir}(1)-P(1)$ & $97.24(5)$ \\
\hline$C(1)-\operatorname{Ir}(1)-P(1)$ & $83.03(5)$ \\
\hline $\mathrm{N}(1)-\operatorname{Ir}(1)-\mathrm{P}(2)$ & $96.93(5)$ \\
\hline $\mathrm{C}(1)-\operatorname{Ir}(1)-\mathrm{P}(2)$ & $82.77(5)$ \\
\hline $\mathrm{P}(1)-\operatorname{Ir}(1)-\mathrm{P}(2)$ & $165.807(18)$ \\
\hline $\mathrm{N}(2)-\mathrm{N}(1)-\operatorname{Ir}(1)$ & $176.0(2)$ \\
\hline $\mathrm{C}(7)-\mathrm{P}(1)-\mathrm{C}(9)$ & $105.67(9)$ \\
\hline $\mathrm{C}(7)-\mathrm{P}(1)-\mathrm{C}(13)$ & $102.77(9)$ \\
\hline $\mathrm{C}(9)-\mathrm{P}(1)-\mathrm{C}(13)$ & $111.23(9)$ \\
\hline $\mathrm{C}(7)-\mathrm{P}(1)-\operatorname{Ir}(1)$ & $104.02(6)$ \\
\hline C(9)-P(1)-Ir(1) & $113.41(6)$ \\
\hline$C(13)-P(1)-\operatorname{Ir}(1)$ & $118.05(7)$ \\
\hline $\mathrm{C}(8)-\mathrm{P}(2)-\mathrm{C}(21)$ & $105.09(9)$ \\
\hline $\mathrm{C}(8)-\mathrm{P}(2)-\mathrm{C}(17)$ & $103.16(10)$ \\
\hline $\mathrm{C}(21)-\mathrm{P}(2)-\mathrm{C}(17)$ & $111.81(9)$ \\
\hline $\mathrm{C}(8)-\mathrm{P}(2)-\operatorname{Ir}(1)$ & $103.96(7)$ \\
\hline $\mathrm{C}(21)-\mathrm{P}(2)-\operatorname{Ir}(1)$ & $112.50(6)$ \\
\hline $\mathrm{C}(17)-\mathrm{P}(2)-\operatorname{Ir}(1)$ & $118.56(7)$ \\
\hline$C(2)-C(1)-C(6)$ & $115.56(17)$ \\
\hline $\mathrm{C}(2)-\mathrm{C}(1)-\operatorname{Ir}(1)$ & $122.25(14)$ \\
\hline$C(6)-C(1)-\operatorname{Ir}(1)$ & $122.09(14)$ \\
\hline $\mathrm{C}(3)-\mathrm{C}(2)-\mathrm{C}(1)$ & $121.93(18)$ \\
\hline $\mathrm{C}(3)-\mathrm{C}(2)-\mathrm{C}(7)$ & $119.03(17)$ \\
\hline$C(1)-C(2)-C(7)$ & $118.97(17)$ \\
\hline$C(4)-C(3)-C(2)$ & $120.57(19)$ \\
\hline $\mathrm{C}(3)-\mathrm{C}(4)-\mathrm{C}(5)$ & $119.24(19)$ \\
\hline$C(4)-C(5)-C(6)$ & $120.67(19)$ \\
\hline$C(5)-C(6)-C(1)$ & $121.93(18)$ \\
\hline $\mathrm{C}(5)-\mathrm{C}(6)-\mathrm{C}(8)$ & $119.48(18)$ \\
\hline $\mathrm{C}(1)-\mathrm{C}(6)-\mathrm{C}(8)$ & $118.56(17)$ \\
\hline $\mathrm{C}(2)-\mathrm{C}(7)-\mathrm{P}(1)$ & $109.23(13)$ \\
\hline $\mathrm{C}(6)-\mathrm{C}(8)-\mathrm{P}(2)$ & $108.77(13)$ \\
\hline $\mathrm{C}(10)-\mathrm{C}(9)-\mathrm{C}(12)$ & $109.54(17)$ \\
\hline $\mathrm{C}(10)-\mathrm{C}(9)-\mathrm{C}(11)$ & $108.93(17)$ \\
\hline $\mathrm{C}(12)-\mathrm{C}(9)-\mathrm{C}(11)$ & $107.51(17)$ \\
\hline $\mathrm{C}(10)-\mathrm{C}(9)-\mathrm{P}(1)$ & $115.06(14)$ \\
\hline $\mathrm{C}(12)-\mathrm{C}(9)-\mathrm{P}(1)$ & $109.17(14)$ \\
\hline $\mathrm{C}(11)-\mathrm{C}(9)-\mathrm{P}(1)$ & $106.34(14)$ \\
\hline$C(14)-C(13)-C(15)$ & $108.16(17)$ \\
\hline $\mathrm{C}(14)-\mathrm{C}(13)-\mathrm{C}(16)$ & $108.98(17)$ \\
\hline $\mathrm{C}(15)-\mathrm{C}(13)-\mathrm{C}(16)$ & $108.70(18)$ \\
\hline $\mathrm{C}(14)-\mathrm{C}(13)-\mathrm{P}(1)$ & $114.53(14)$ \\
\hline $\mathrm{C}(15)-\mathrm{C}(13)-\mathrm{P}(1)$ & $105.35(14)$ \\
\hline $\mathrm{C}(16)-\mathrm{C}(13)-\mathrm{P}(1)$ & $110.89(14)$ \\
\hline$C(20)-C(17)-C(19)$ & $109.16(19)$ \\
\hline $\mathrm{C}(20)-\mathrm{C}(17)-\mathrm{C}(18)$ & $108.7(2)$ \\
\hline$C(19)-C(17)-C(18)$ & $108.1(2)$ \\
\hline $\mathrm{C}(20)-\mathrm{C}(17)-\mathrm{P}(2)$ & $110.82(15)$ \\
\hline $\mathrm{C}(19)-\mathrm{C}(17)-\mathrm{P}(2)$ & $114.48(16)$ \\
\hline $\mathrm{C}(18)-\mathrm{C}(17)-\mathrm{P}(2)$ & $105.39(15)$ \\
\hline $\mathrm{C}(23)-\mathrm{C}(21)-\mathrm{C}(24)$ & $109.59(17)$ \\
\hline $\mathrm{C}(23)-\mathrm{C}(21)-\mathrm{C}(22)$ & $109.51(17)$ \\
\hline $\mathrm{C}(24)-\mathrm{C}(21)-\mathrm{C}(22)$ & $107.78(17)$ \\
\hline $\mathrm{C}(23)-\mathrm{C}(21)-\mathrm{P}(2)$ & $114.69(14)$ \\
\hline $\mathrm{C}(24)-\mathrm{C}(21)-\mathrm{P}(2)$ & $108.92(14)$ \\
\hline $\mathrm{C}(22)-\mathrm{C}(21)-\mathrm{P}(2)$ & $106.09(13)$ \\
\hline$N(3)-\operatorname{Ir}(2)-C(25)$ & $179.79(6)$ \\
\hline $\mathrm{N}(3)-\operatorname{Ir}(2)-\mathrm{P}(4)$ & $97.20(5)$ \\
\hline $\mathrm{C}(25)-\operatorname{Ir}(2)-\mathrm{P}(4)$ & $82.98(5)$ \\
\hline $\mathrm{N}(3)-\operatorname{Ir}(2)-\mathrm{P}(3)$ & $97.38(5)$ \\
\hline $\mathrm{C}(25)-\operatorname{Ir}(2)-\mathrm{P}(3)$ & $82.45(5)$ \\
\hline$P(4)-\operatorname{Ir}(2)-P(3)$ & $165.110(17)$ \\
\hline
\end{tabular}

\begin{tabular}{|c|c|}
\hline$N(4)-N(3)-\operatorname{Ir}(2)$ & $179.20(19)$ \\
\hline $\mathrm{C}(31)-\mathrm{P}(3)-\mathrm{C}(37)$ & $104.95(9)$ \\
\hline $\mathrm{C}(31)-\mathrm{P}(3)-\mathrm{C}(33)$ & $102.75(9)$ \\
\hline $\mathrm{C}(37)-\mathrm{P}(3)-\mathrm{C}(33)$ & $112.30(9)$ \\
\hline $\mathrm{C}(31)-\mathrm{P}(3)-\operatorname{Ir}(2)$ & $104.46(6)$ \\
\hline $\mathrm{C}(37)-\mathrm{P}(3)-\operatorname{Ir}(2)$ & $111.37(6)$ \\
\hline $\mathrm{C}(33)-\mathrm{P}(3)-\operatorname{Ir}(2)$ & $119.24(6)$ \\
\hline $\mathrm{C}(32)-\mathrm{P}(4)-\mathrm{C}(41)$ & $104.77(9)$ \\
\hline $\mathrm{C}(32)-\mathrm{P}(4)-\mathrm{C}(45)$ & $103.07(9)$ \\
\hline $\mathrm{C}(41)-\mathrm{P}(4)-\mathrm{C}(45)$ & $111.11(9)$ \\
\hline $\mathrm{C}(32)-\mathrm{P}(4)-\operatorname{Ir}(2)$ & $104.41(6)$ \\
\hline $\mathrm{C}(41)-\mathrm{P}(4)-\operatorname{Ir}(2)$ & $114.36(7)$ \\
\hline $\mathrm{C}(45)-\mathrm{P}(4)-\operatorname{Ir}(2)$ & $117.34(6)$ \\
\hline$C(30)-C(25)-C(26)$ & $115.91(16)$ \\
\hline$C(30)-C(25)-\operatorname{Ir}(2)$ & $122.06(14)$ \\
\hline$C(26)-C(25)-\operatorname{Ir}(2)$ & $122.03(13)$ \\
\hline$C(27)-C(26)-C(25)$ & $121.92(17)$ \\
\hline $\mathrm{C}(27)-\mathrm{C}(26)-\mathrm{C}(31)$ & $118.79(17)$ \\
\hline$C(25)-C(26)-C(31)$ & $119.24(16)$ \\
\hline $\mathrm{C}(28)-\mathrm{C}(27)-\mathrm{C}(26)$ & $120.54(19)$ \\
\hline $\mathrm{C}(27)-\mathrm{C}(28)-\mathrm{C}(29)$ & $119.42(18)$ \\
\hline $\mathrm{C}(28)-\mathrm{C}(29)-\mathrm{C}(30)$ & $120.71(18)$ \\
\hline $\mathrm{C}(29)-\mathrm{C}(30)-\mathrm{C}(25)$ & $121.43(18)$ \\
\hline$C(29)-C(30)-C(32)$ & $119.31(17)$ \\
\hline $\mathrm{C}(25)-\mathrm{C}(30)-\mathrm{C}(32)$ & $119.19(16)$ \\
\hline $\mathrm{C}(26)-\mathrm{C}(31)-\mathrm{P}(3)$ & $108.86(13)$ \\
\hline $\mathrm{C}(30)-\mathrm{C}(32)-\mathrm{P}(4)$ & $109.47(13)$ \\
\hline$C(36)-C(33)-C(34)$ & $109.28(16)$ \\
\hline $\mathrm{C}(36)-\mathrm{C}(33)-\mathrm{C}(35)$ & $108.29(17)$ \\
\hline $\mathrm{C}(34)-\mathrm{C}(33)-\mathrm{C}(35)$ & $108.62(17)$ \\
\hline $\mathrm{C}(36)-\mathrm{C}(33)-\mathrm{P}(3)$ & $111.05(14)$ \\
\hline $\mathrm{C}(34)-\mathrm{C}(33)-\mathrm{P}(3)$ & $114.17(14)$ \\
\hline C(35)-C(33)-P(3) & $105.18(13)$ \\
\hline $\mathrm{C}(38)-\mathrm{C}(37)-\mathrm{C}(40)$ & $109.81(16)$ \\
\hline $\mathrm{C}(38)-\mathrm{C}(37)-\mathrm{C}(39)$ & $108.77(17)$ \\
\hline $\mathrm{C}(40)-\mathrm{C}(37)-\mathrm{C}(39)$ & $107.59(17)$ \\
\hline $\mathrm{C}(38)-\mathrm{C}(37)-\mathrm{P}(3)$ & $115.09(14)$ \\
\hline $\mathrm{C}(40)-\mathrm{C}(37)-\mathrm{P}(3)$ & $108.77(13)$ \\
\hline $\mathrm{C}(39)-\mathrm{C}(37)-\mathrm{P}(3)$ & $106.52(13)$ \\
\hline $\mathrm{C}(43)-\mathrm{C}(41)-\mathrm{C}(44)$ & $109.49(18)$ \\
\hline $\mathrm{C}(43)-\mathrm{C}(41)-\mathrm{C}(42)$ & $109.43(18)$ \\
\hline $\mathrm{C}(44)-\mathrm{C}(41)-\mathrm{C}(42)$ & $107.32(18)$ \\
\hline $\mathrm{C}(43)-\mathrm{C}(41)-\mathrm{P}(4)$ & $114.65(14)$ \\
\hline $\mathrm{C}(44)-\mathrm{C}(41)-\mathrm{P}(4)$ & $109.71(14)$ \\
\hline $\mathrm{C}(42)-\mathrm{C}(41)-\mathrm{P}(4)$ & $105.96(15)$ \\
\hline$C(46)-C(45)-C(47)$ & $108.26(17)$ \\
\hline$C(46)-C(45)-C(48)$ & $108.46(17)$ \\
\hline $\mathrm{C}(47)-\mathrm{C}(45)-\mathrm{C}(48)$ & $108.95(17)$ \\
\hline $\mathrm{C}(46)-\mathrm{C}(45)-\mathrm{P}(4)$ & $105.30(14)$ \\
\hline $\mathrm{C}(47)-\mathrm{C}(45)-\mathrm{P}(4)$ & $115.04(14)$ \\
\hline $\mathrm{C}(48)-\mathrm{C}(45)-\mathrm{P}(4)$ & $110.58(14)$ \\
\hline$N(5)-\operatorname{Ir}(3)-C(49)$ & $179.14(7)$ \\
\hline $\mathrm{N}(5)-\operatorname{Ir}(3)-\mathrm{P}(6)$ & $97.16(5)$ \\
\hline$C(49)-\operatorname{Ir}(3)-P(6)$ & $82.63(5)$ \\
\hline $\mathrm{N}(5)-\operatorname{Ir}(3)-\mathrm{P}(5)$ & $97.47(5)$ \\
\hline$C(49)-\operatorname{Ir}(3)-P(5)$ & $82.74(5)$ \\
\hline$P(6)-\operatorname{Ir}(3)-P(5)$ & $165.370(17)$ \\
\hline$N(6)-N(5)-\operatorname{Ir}(3)$ & $179.63(18)$ \\
\hline $\mathrm{C}(55)-\mathrm{P}(5)-\mathrm{C}(61)$ & $103.36(9)$ \\
\hline $\mathrm{C}(55)-\mathrm{P}(5)-\mathrm{C}(57)$ & $104.87(9)$ \\
\hline $\mathrm{C}(61)-\mathrm{P}(5)-\mathrm{C}(57)$ & $111.46(9)$ \\
\hline $\mathrm{C}(55)-\mathrm{P}(5)-\operatorname{Ir}(3)$ & $104.07(6)$ \\
\hline $\mathrm{C}(61)-\mathrm{P}(5)-\operatorname{Ir}(3)$ & $118.57(7)$ \\
\hline
\end{tabular}




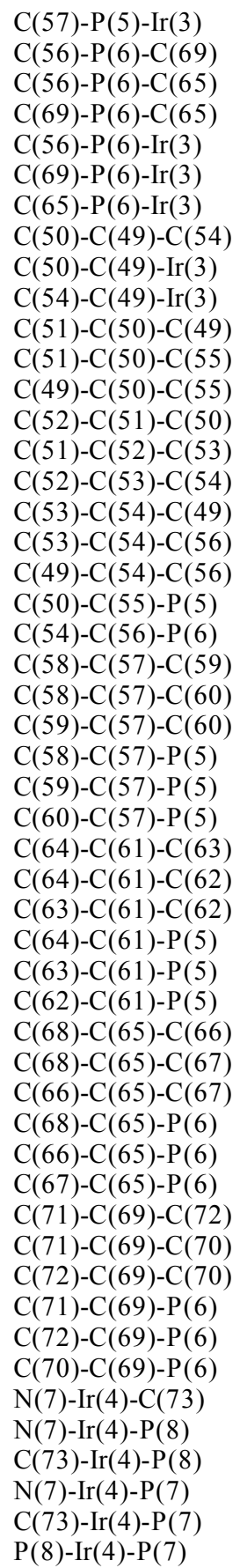

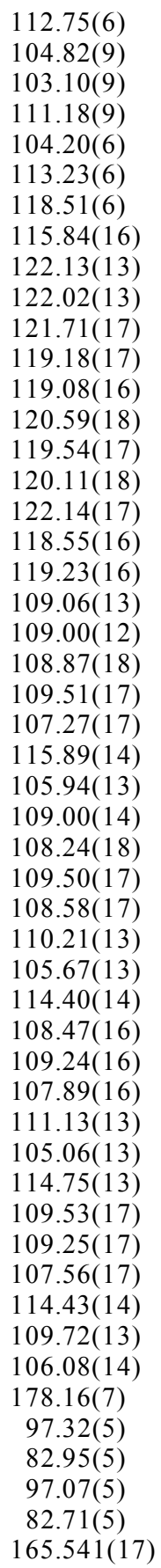

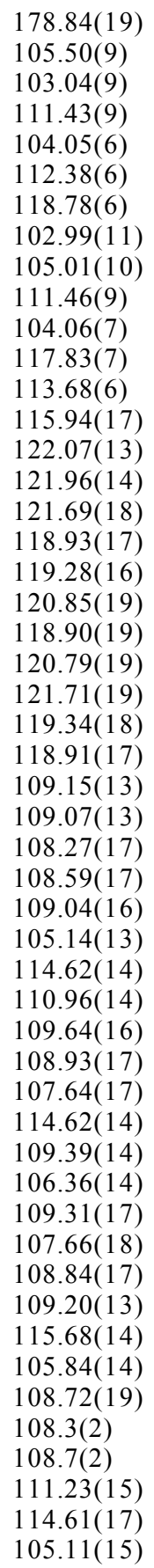


Table S-4. Torsion angles $\left[{ }^{\circ}\right]$ for complex 1.

\begin{tabular}{|c|c|c|c|}
\hline $\mathrm{C}(1)-\operatorname{Ir}(1)-\mathrm{N}(1)-\mathrm{N}(2)$ & $2(3)$ & $\operatorname{Ir}(1)-\mathrm{P}(1)-\mathrm{C}(13)-\mathrm{C}(14)$ & $-157.50(13)$ \\
\hline $\mathrm{P}(1)-\operatorname{Ir}(1)-\mathrm{N}(1)-\mathrm{N}(2)$ & $95(3)$ & $\mathrm{C}(7)-\mathrm{P}(1)-\mathrm{C}(13)-\mathrm{C}(15)$ & 74.94(16) \\
\hline $\mathrm{P}(2)-\operatorname{Ir}(1)-\mathrm{N}(1)-\mathrm{N}(2)$ & $-84(3)$ & $\mathrm{C}(9)-\mathrm{P}(1)-\mathrm{C}(13)-\mathrm{C}(15)$ & $-172.41(14)$ \\
\hline $\mathrm{N}(1)-\operatorname{Ir}(1)-\mathrm{P}(1)-\mathrm{C}(7)$ & $-162.88(9)$ & $\operatorname{Ir}(1)-\mathrm{P}(1)-\mathrm{C}(13)-\mathrm{C}(15)$ & $-38.78(16)$ \\
\hline $\mathrm{C}(1)-\operatorname{Ir}(1)-\mathrm{P}(1)-\mathrm{C}(7)$ & 12.61(9) & $\mathrm{C}(7)-\mathrm{P}(1)-\mathrm{C}(13)-\mathrm{C}(16)$ & $-167.62(15)$ \\
\hline$P(2)-\operatorname{Ir}(1)-P(1)-C(7)$ & $13.57(11)$ & $\mathrm{C}(9)-\mathrm{P}(1)-\mathrm{C}(13)-\mathrm{C}(16)$ & $-54.97(17)$ \\
\hline $\mathrm{N}(1)-\operatorname{Ir}(1)-\mathrm{P}(1)-\mathrm{C}(9)$ & $82.83(9)$ & $\operatorname{Ir}(1)-\mathrm{P}(1)-\mathrm{C}(13)-\mathrm{C}(16)$ & $78.66(15)$ \\
\hline $\mathrm{C}(1)-\operatorname{Ir}(1)-\mathrm{P}(1)-\mathrm{C}(9)$ & $-101.69(9)$ & $\mathrm{C}(8)-\mathrm{P}(2)-\mathrm{C}(17)-\mathrm{C}(20)$ & $-170.61(16)$ \\
\hline $\mathrm{P}(2)-\operatorname{Ir}(1)-\mathrm{P}(1)-\mathrm{C}(9)$ & $-100.73(10)$ & $\mathrm{C}(21)-\mathrm{P}(2)-\mathrm{C}(17)-\mathrm{C}(20)$ & $-58.19(18)$ \\
\hline $\mathrm{N}(1)-\operatorname{Ir}(1)-\mathrm{P}(1)-\mathrm{C}(13)$ & $-49.85(10)$ & $\operatorname{Ir}(1)-P(2)-C(17)-C(20)$ & $75.22(17)$ \\
\hline $\mathrm{C}(1)-\operatorname{Ir}(1)-\mathrm{P}(1)-\mathrm{C}(13)$ & $125.64(9)$ & $\mathrm{C}(8)-\mathrm{P}(2)-\mathrm{C}(17)-\mathrm{C}(19)$ & $-46.64(18)$ \\
\hline $\mathrm{P}(2)-\operatorname{Ir}(1)-\mathrm{P}(1)-\mathrm{C}(13)$ & $126.60(10)$ & $\mathrm{C}(21)-\mathrm{P}(2)-\mathrm{C}(17)-\mathrm{C}(19)$ & $65.78(19)$ \\
\hline $\mathrm{N}(1)-\operatorname{Ir}(1)-\mathrm{P}(2)-\mathrm{C}(8)$ & $-171.44(9)$ & $\operatorname{Ir}(1)-P(2)-C(17)-C(19)$ & $-160.81(14)$ \\
\hline $\mathrm{C}(1)-\operatorname{Ir}(1)-\mathrm{P}(2)-\mathrm{C}(8)$ & $13.07(9)$ & $\mathrm{C}(8)-\mathrm{P}(2)-\mathrm{C}(17)-\mathrm{C}(18)$ & $72.02(19)$ \\
\hline $\mathrm{P}(1)-\operatorname{Ir}(1)-\mathrm{P}(2)-\mathrm{C}(8)$ & $12.11(11)$ & $\mathrm{C}(21)-\mathrm{P}(2)-\mathrm{C}(17)-\mathrm{C}(18)$ & $-175.55(17)$ \\
\hline$N(1)-\operatorname{Ir}(1)-P(2)-C(21)$ & $75.41(9)$ & $\operatorname{Ir}(1)-P(2)-C(17)-C(18)$ & $-42.1(2)$ \\
\hline $\mathrm{C}(1)-\operatorname{Ir}(1)-\mathrm{P}(2)-\mathrm{C}(21)$ & $-100.08(9)$ & $\mathrm{C}(8)-\mathrm{P}(2)-\mathrm{C}(21)-\mathrm{C}(23)$ & $73.81(17)$ \\
\hline $\mathrm{P}(1)-\operatorname{Ir}(1)-\mathrm{P}(2)-\mathrm{C}(21)$ & $-101.04(10)$ & $\mathrm{C}(17)-\mathrm{P}(2)-\mathrm{C}(21)-\mathrm{C}(23)$ & $-37.41(18)$ \\
\hline$N(1)-\operatorname{Ir}(1)-P(2)-C(17)$ & $-57.71(10)$ & $\operatorname{Ir}(1)-P(2)-C(21)-C(23)$ & $-173.73(13)$ \\
\hline $\mathrm{C}(1)-\operatorname{Ir}(1)-\mathrm{P}(2)-\mathrm{C}(17)$ & $126.80(10)$ & $\mathrm{C}(8)-\mathrm{P}(2)-\mathrm{C}(21)-\mathrm{C}(24)$ & $-162.99(14)$ \\
\hline $\mathrm{P}(1)-\operatorname{Ir}(1)-\mathrm{P}(2)-\mathrm{C}(17)$ & $125.85(10)$ & $\mathrm{C}(17)-\mathrm{P}(2)-\mathrm{C}(21)-\mathrm{C}(24)$ & $85.80(16)$ \\
\hline $\mathrm{N}(1)-\operatorname{Ir}(1)-\mathrm{C}(1)-\mathrm{C}(2)$ & $84.8(10)$ & $\operatorname{Ir}(1)-P(2)-C(21)-C(24)$ & $-50.52(15)$ \\
\hline $\mathrm{P}(1)-\operatorname{Ir}(1)-\mathrm{C}(1)-\mathrm{C}(2)$ & $-9.02(14)$ & $\mathrm{C}(8)-\mathrm{P}(2)-\mathrm{C}(21)-\mathrm{C}(22)$ & $-47.21(16)$ \\
\hline$P(2)-\operatorname{Ir}(1)-C(1)-C(2)$ & $171.22(15)$ & $\mathrm{C}(17)-\mathrm{P}(2)-\mathrm{C}(21)-\mathrm{C}(22)$ & $-158.42(14)$ \\
\hline $\mathrm{N}(1)-\operatorname{Ir}(1)-\mathrm{C}(1)-\mathrm{C}(6)$ & $-91.5(10)$ & $\operatorname{Ir}(1)-P(2)-C(21)-C(22)$ & $65.26(14)$ \\
\hline $\mathrm{P}(1)-\operatorname{Ir}(1)-\mathrm{C}(1)-\mathrm{C}(6)$ & $174.74(15)$ & $\mathrm{C}(25)-\operatorname{Ir}(2)-\mathrm{N}(3)-\mathrm{N}(4)$ & $114(29)$ \\
\hline$P(2)-\operatorname{Ir}(1)-C(1)-C(6)$ & $-5.02(14)$ & $\mathrm{P}(4)-\operatorname{Ir}(2)-\mathrm{N}(3)-\mathrm{N}(4)$ & $-98(13)$ \\
\hline$C(6)-C(1)-C(2)-C(3)$ & $-0.3(3)$ & $\mathrm{P}(3)-\operatorname{Ir}(2)-\mathrm{N}(3)-\mathrm{N}(4)$ & $78(13)$ \\
\hline $\operatorname{Ir}(1)-C(1)-C(2)-C(3)$ & $-176.74(15)$ & $\mathrm{N}(3)-\operatorname{Ir}(2)-\mathrm{P}(3)-\mathrm{C}(31)$ & $167.14(9)$ \\
\hline$C(6)-C(1)-C(2)-C(7)$ & $176.73(17)$ & $C(25)-\operatorname{Ir}(2)-P(3)-C(31)$ & $-12.74(8)$ \\
\hline $\operatorname{Ir}(1)-C(1)-C(2)-C(7)$ & $0.3(2)$ & $P(4)-\operatorname{Ir}(2)-P(3)-C(31)$ & $-24.79(10)$ \\
\hline$C(1)-C(2)-C(3)-C(4)$ & $2.5(3)$ & $\mathrm{N}(3)-\operatorname{Ir}(2)-\mathrm{P}(3)-\mathrm{C}(37)$ & $-80.09(9)$ \\
\hline$C(7)-C(2)-C(3)-C(4)$ & $-174.54(19)$ & $\mathrm{C}(25)-\operatorname{Ir}(2)-\mathrm{P}(3)-\mathrm{C}(37)$ & $100.03(8)$ \\
\hline $\mathrm{C}(2)-\mathrm{C}(3)-\mathrm{C}(4)-\mathrm{C}(5)$ & $-1.8(3)$ & $\mathrm{P}(4)-\operatorname{Ir}(2)-\mathrm{P}(3)-\mathrm{C}(37)$ & $87.98(9)$ \\
\hline$C(3)-C(4)-C(5)-C(6)$ & $-1.0(3)$ & $\mathrm{N}(3)-\operatorname{Ir}(2)-\mathrm{P}(3)-\mathrm{C}(33)$ & $53.22(9)$ \\
\hline$C(4)-C(5)-C(6)-C(1)$ & $3.3(3)$ & $\mathrm{C}(25)-\operatorname{Ir}(2)-\mathrm{P}(3)-\mathrm{C}(33)$ & $-126.66(9)$ \\
\hline $\mathrm{C}(4)-\mathrm{C}(5)-\mathrm{C}(6)-\mathrm{C}(8)$ & $-174.87(19)$ & $\mathrm{P}(4)-\operatorname{Ir}(2)-\mathrm{P}(3)-\mathrm{C}(33)$ & $-138.71(9)$ \\
\hline$C(2)-C(1)-C(6)-C(5)$ & $-2.6(3)$ & $\mathrm{N}(3)-\operatorname{Ir}(2)-\mathrm{P}(4)-\mathrm{C}(32)$ & $170.50(9)$ \\
\hline $\operatorname{Ir}(1)-C(1)-C(6)-C(5)$ & $173.90(15)$ & $\mathrm{C}(25)-\operatorname{Ir}(2)-\mathrm{P}(4)-\mathrm{C}(32)$ & $-9.61(9)$ \\
\hline $\mathrm{C}(2)-\mathrm{C}(1)-\mathrm{C}(6)-\mathrm{C}(8)$ & $175.62(17)$ & $\mathrm{P}(3)-\operatorname{Ir}(2)-\mathrm{P}(4)-\mathrm{C}(32)$ & $2.43(11)$ \\
\hline $\operatorname{Ir}(1)-C(1)-C(6)-C(8)$ & $-7.9(2)$ & $\mathrm{N}(3)-\operatorname{Ir}(2)-\mathrm{P}(4)-\mathrm{C}(41)$ & $-75.59(9)$ \\
\hline$C(3)-C(2)-C(7)-P(1)$ & $-171.54(15)$ & $C(25)-\operatorname{Ir}(2)-P(4)-C(41)$ & $104.30(9)$ \\
\hline $\mathrm{C}(1)-\mathrm{C}(2)-\mathrm{C}(7)-\mathrm{P}(1)$ & $11.4(2)$ & $\mathrm{P}(3)-\operatorname{Ir}(2)-\mathrm{P}(4)-\mathrm{C}(41)$ & $116.34(10)$ \\
\hline $\mathrm{C}(9)-\mathrm{P}(1)-\mathrm{C}(7)-\mathrm{C}(2)$ & $103.87(14)$ & $\mathrm{N}(3)-\operatorname{Ir}(2)-\mathrm{P}(4)-\mathrm{C}(45)$ & $57.19(9)$ \\
\hline $\mathrm{C}(13)-\mathrm{P}(1)-\mathrm{C}(7)-\mathrm{C}(2)$ & $-139.44(14)$ & $C(25)-\operatorname{Ir}(2)-P(4)-C(45)$ & $-122.92(9)$ \\
\hline $\operatorname{Ir}(1)-P(1)-C(7)-C(2)$ & $-15.83(15)$ & $\mathrm{P}(3)-\operatorname{Ir}(2)-\mathrm{P}(4)-\mathrm{C}(45)$ & $-110.88(9)$ \\
\hline $\mathrm{C}(5)-\mathrm{C}(6)-\mathrm{C}(8)-\mathrm{P}(2)$ & $-162.72(16)$ & $\mathrm{N}(3)-\operatorname{Ir}(2)-\mathrm{C}(25)-\mathrm{C}(30)$ & $152(100)$ \\
\hline $\mathrm{C}(1)-\mathrm{C}(6)-\mathrm{C}(8)-\mathrm{P}(2)$ & $19.0(2)$ & $\mathrm{P}(4)-\operatorname{Ir}(2)-\mathrm{C}(25)-\mathrm{C}(30)$ & $4.45(14)$ \\
\hline $\mathrm{C}(21)-\mathrm{P}(2)-\mathrm{C}(8)-\mathrm{C}(6)$ & $98.38(15)$ & $\mathrm{P}(3)-\operatorname{Ir}(2)-\mathrm{C}(25)-\mathrm{C}(30)$ & $-172.46(15)$ \\
\hline $\mathrm{C}(17)-\mathrm{P}(2)-\mathrm{C}(8)-\mathrm{C}(6)$ & $-144.34(14)$ & $\mathrm{N}(3)-\operatorname{Ir}(2)-\mathrm{C}(25)-\mathrm{C}(26)$ & $-28(26)$ \\
\hline $\operatorname{Ir}(1)-P(2)-C(8)-C(6)$ & $-20.00(15)$ & $P(4)-\operatorname{Ir}(2)-C(25)-C(26)$ & $-176.13(15)$ \\
\hline $\mathrm{C}(7)-\mathrm{P}(1)-\mathrm{C}(9)-\mathrm{C}(10)$ & $69.55(17)$ & $\mathrm{P}(3)-\operatorname{Ir}(2)-\mathrm{C}(25)-\mathrm{C}(26)$ & $6.97(14)$ \\
\hline$C(13)-P(1)-C(9)-C(10)$ & $-41.25(18)$ & $\mathrm{C}(30)-\mathrm{C}(25)-\mathrm{C}(26)-\mathrm{C}(27)$ & $0.5(3)$ \\
\hline $\operatorname{Ir}(1)-P(1)-C(9)-C(10)$ & $-177.14(13)$ & $\operatorname{Ir}(2)-C(25)-C(26)-C(27)$ & $-178.95(14)$ \\
\hline $\mathrm{C}(7)-\mathrm{P}(1)-\mathrm{C}(9)-\mathrm{C}(12)$ & $-166.84(14)$ & $\mathrm{C}(30)-\mathrm{C}(25)-\mathrm{C}(26)-\mathrm{C}(31)$ & $-176.84(17)$ \\
\hline$C(13)-P(1)-C(9)-C(12)$ & $82.36(16)$ & $\operatorname{Ir}(2)-C(25)-C(26)-C(31)$ & $3.7(2)$ \\
\hline $\operatorname{Ir}(1)-P(1)-C(9)-C(12)$ & $-53.53(15)$ & $\mathrm{C}(25)-\mathrm{C}(26)-\mathrm{C}(27)-\mathrm{C}(28)$ & $-2.4(3)$ \\
\hline $\mathrm{C}(7)-\mathrm{P}(1)-\mathrm{C}(9)-\mathrm{C}(11)$ & $-51.12(16)$ & $\mathrm{C}(31)-\mathrm{C}(26)-\mathrm{C}(27)-\mathrm{C}(28)$ & $174.99(18)$ \\
\hline $\mathrm{C}(13)-\mathrm{P}(1)-\mathrm{C}(9)-\mathrm{C}(11)$ & $-161.93(14)$ & $\mathrm{C}(26)-\mathrm{C}(27)-\mathrm{C}(28)-\mathrm{C}(29)$ & $1.8(3)$ \\
\hline $\operatorname{Ir}(1)-P(1)-C(9)-C(11)$ & $62.19(15)$ & $\mathrm{C}(27)-\mathrm{C}(28)-\mathrm{C}(29)-\mathrm{C}(30)$ & $0.5(3)$ \\
\hline $\mathrm{C}(7)-\mathrm{P}(1)-\mathrm{C}(13)-\mathrm{C}(14)$ & $-43.78(17)$ & $\mathrm{C}(28)-\mathrm{C}(29)-\mathrm{C}(30)-\mathrm{C}(25)$ & $-2.4(3)$ \\
\hline $\mathrm{C}(9)-\mathrm{P}(1)-\mathrm{C}(13)-\mathrm{C}(14)$ & $68.87(17)$ & $\mathrm{C}(28)-\mathrm{C}(29)-\mathrm{C}(30)-\mathrm{C}(32)$ & $174.44(19)$ \\
\hline
\end{tabular}


$\mathrm{C}(26)-\mathrm{C}(25)-\mathrm{C}(30)-\mathrm{C}(29)$ $\operatorname{Ir}(2)-\mathrm{C}(25)-\mathrm{C}(30)-\mathrm{C}(29)$ $\mathrm{C}(26)-\mathrm{C}(25)-\mathrm{C}(30)-\mathrm{C}(32)$ $\operatorname{Ir}(2)-\mathrm{C}(25)-\mathrm{C}(30)-\mathrm{C}(32)$ $\mathrm{C}(27)-\mathrm{C}(26)-\mathrm{C}(31)-\mathrm{P}(3)$ $\mathrm{C}(25)-\mathrm{C}(26)-\mathrm{C}(31)-\mathrm{P}(3)$ $\mathrm{C}(37)-\mathrm{P}(3)-\mathrm{C}(31)-\mathrm{C}(26)$ $\mathrm{C}(33)-\mathrm{P}(3)-\mathrm{C}(31)-\mathrm{C}(26)$ $\operatorname{Ir}(2)-\mathrm{P}(3)-\mathrm{C}(31)-\mathrm{C}(26)$ $\mathrm{C}(29)-\mathrm{C}(30)-\mathrm{C}(32)-\mathrm{P}(4)$ $\mathrm{C}(25)-\mathrm{C}(30)-\mathrm{C}(32)-\mathrm{P}(4)$ $\mathrm{C}(41)-\mathrm{P}(4)-\mathrm{C}(32)-\mathrm{C}(30)$ $\mathrm{C}(45)-\mathrm{P}(4)-\mathrm{C}(32)-\mathrm{C}(30)$ $\operatorname{Ir}(2)-\mathrm{P}(4)-\mathrm{C}(32)-\mathrm{C}(30)$ $\mathrm{C}(31)-\mathrm{P}(3)-\mathrm{C}(33)-\mathrm{C}(36)$ $\mathrm{C}(37)-\mathrm{P}(3)-\mathrm{C}(33)-\mathrm{C}(36)$ $\operatorname{Ir}(2)-\mathrm{P}(3)-\mathrm{C}(33)-\mathrm{C}(36)$ $\mathrm{C}(31)-\mathrm{P}(3)-\mathrm{C}(33)-\mathrm{C}(34)$ $\mathrm{C}(37)-\mathrm{P}(3)-\mathrm{C}(33)-\mathrm{C}(34)$ $\operatorname{Ir}(2)-\mathrm{P}(3)-\mathrm{C}(33)-\mathrm{C}(34)$ $\mathrm{C}(31)-\mathrm{P}(3)-\mathrm{C}(33)-\mathrm{C}(35)$ $\mathrm{C}(37)-\mathrm{P}(3)-\mathrm{C}(33)-\mathrm{C}(35)$ $\operatorname{Ir}(2)-\mathrm{P}(3)-\mathrm{C}(33)-\mathrm{C}(35)$ $\mathrm{C}(31)-\mathrm{P}(3)-\mathrm{C}(37)-\mathrm{C}(38)$ $\mathrm{C}(33)-\mathrm{P}(3)-\mathrm{C}(37)-\mathrm{C}(38)$ $\operatorname{Ir}(2)-\mathrm{P}(3)-\mathrm{C}(37)-\mathrm{C}(38)$ $\mathrm{C}(31)-\mathrm{P}(3)-\mathrm{C}(37)-\mathrm{C}(40)$ $\mathrm{C}(33)-\mathrm{P}(3)-\mathrm{C}(37)-\mathrm{C}(40)$ $\operatorname{Ir}(2)-\mathrm{P}(3)-\mathrm{C}(37)-\mathrm{C}(40)$ $\mathrm{C}(31)-\mathrm{P}(3)-\mathrm{C}(37)-\mathrm{C}(39)$ $\mathrm{C}(33)-\mathrm{P}(3)-\mathrm{C}(37)-\mathrm{C}(39)$ $\operatorname{Ir}(2)-\mathrm{P}(3)-\mathrm{C}(37)-\mathrm{C}(39)$ $\mathrm{C}(32)-\mathrm{P}(4)-\mathrm{C}(41)-\mathrm{C}(43)$ C(45)-P(4)-C(41)-C(43) $\operatorname{Ir}(2)-\mathrm{P}(4)-\mathrm{C}(41)-\mathrm{C}(43)$ $\mathrm{C}(32)-\mathrm{P}(4)-\mathrm{C}(41)-\mathrm{C}(44)$ $\mathrm{C}(45)-\mathrm{P}(4)-\mathrm{C}(41)-\mathrm{C}(44)$ $\operatorname{Ir}(2)-\mathrm{P}(4)-\mathrm{C}(41)-\mathrm{C}(44)$ $\mathrm{C}(32)-\mathrm{P}(4)-\mathrm{C}(41)-\mathrm{C}(42)$ $\mathrm{C}(45)-\mathrm{P}(4)-\mathrm{C}(41)-\mathrm{C}(42)$ $\operatorname{Ir}(2)-\mathrm{P}(4)-\mathrm{C}(41)-\mathrm{C}(42)$ $\mathrm{C}(32)-\mathrm{P}(4)-\mathrm{C}(45)-\mathrm{C}(46)$ $\mathrm{C}(41)-\mathrm{P}(4)-\mathrm{C}(45)-\mathrm{C}(46)$ $\operatorname{Ir}(2)-\mathrm{P}(4)-\mathrm{C}(45)-\mathrm{C}(46)$ $\mathrm{C}(32)-\mathrm{P}(4)-\mathrm{C}(45)-\mathrm{C}(47)$ $\mathrm{C}(41)-\mathrm{P}(4)-\mathrm{C}(45)-\mathrm{C}(47)$ $\operatorname{Ir}(2)-\mathrm{P}(4)-\mathrm{C}(45)-\mathrm{C}(47)$ $\mathrm{C}(32)-\mathrm{P}(4)-\mathrm{C}(45)-\mathrm{C}(48)$ $\mathrm{C}(41)-\mathrm{P}(4)-\mathrm{C}(45)-\mathrm{C}(48)$ $\operatorname{Ir}(2)-\mathrm{P}(4)-\mathrm{C}(45)-\mathrm{C}(48)$ $\mathrm{C}(49)-\operatorname{Ir}(3)-\mathrm{N}(5)-\mathrm{N}(6)$ $\mathrm{P}(6)-\operatorname{Ir}(3)-\mathrm{N}(5)-\mathrm{N}(6)$ $\mathrm{P}(5)-\operatorname{Ir}(3)-\mathrm{N}(5)-\mathrm{N}(6)$ $\mathrm{N}(5)-\operatorname{Ir}(3)-\mathrm{P}(5)-\mathrm{C}(55)$ $\mathrm{C}(49)-\operatorname{Ir}(3)-\mathrm{P}(5)-\mathrm{C}(55)$ $\mathrm{P}(6)-\operatorname{Ir}(3)-\mathrm{P}(5)-\mathrm{C}(55)$ $\mathrm{N}(5)-\operatorname{Ir}(3)-\mathrm{P}(5)-\mathrm{C}(61)$ $\mathrm{C}(49)-\operatorname{Ir}(3)-\mathrm{P}(5)-\mathrm{C}(61)$ $\mathrm{P}(6)-\operatorname{Ir}(3)-\mathrm{P}(5)-\mathrm{C}(61)$ $\mathrm{N}(5)-\operatorname{Ir}(3)-\mathrm{P}(5)-\mathrm{C}(57)$ $\mathrm{C}(49)-\operatorname{Ir}(3)-\mathrm{P}(5)-\mathrm{C}(57)$ $\mathrm{P}(6)-\operatorname{Ir}(3)-\mathrm{P}(5)-\mathrm{C}(57)$ $\mathrm{N}(5)-\operatorname{Ir}(3)-\mathrm{P}(6)-\mathrm{C}(56)$ $\mathrm{C}(49)-\operatorname{Ir}(3)-\mathrm{P}(6)-\mathrm{C}(56)$

\footnotetext{
$1.9(3)$

$-178.68(14)$

$-175.00(17)$ $4.5(2)$

$167.60(15)$

$-15.0(2)$

$-99.57(14)$

$142.85(13)$

$17.71(14)$

$170.25(15)$

$-12.8(2)$

$-106.39(15)$

$137.28(14)$

$14.16(15)$

$172.15(15)$

59.90(17)

$-73.01(15)$

48.06(16)

$-64.19(16)$

$162.90(12)$

$-70.92(15)$

176.83(13)

43.92(15)

$-72.56(16)$

$38.31(17)$

$174.98(13)$

163.77(14)

$-85.35(15)$

$51.32(14)$

48.06(15)

158.94(13)

$-64.40(14)$

$-67.40(17)$

43.23(18)

$178.90(13)$

168.94(15)

$-80.43(17)$

55.24(17)

$53.39(16)$

164.03(14)

$-60.31(15)$

$-72.98(15)$

175.29(13)

$41.08(15)$

46.12(16)

$-65.60(17)$

$160.18(12)$

$170.06(15)$

$58.34(16)$

$-75.88(15)$

22(31)

98(29)

$-82(29)$

$-168.65(8)$

12.19(8)

$12.33(10)$

$-54.59(9)$

126.25(9)

126.39(9)

78.26(9)

$-100.90(9)$

$-100.76(9)$

$-166.41(8)$

$12.76(8)$
}

$\mathrm{P}(5)-\operatorname{Ir}(3)-\mathrm{P}(6)-\mathrm{C}(56)$

$\mathrm{N}(5)-\operatorname{Ir}(3)-\mathrm{P}(6)-\mathrm{C}(69)$

$\mathrm{C}(49)-\operatorname{Ir}(3)-\mathrm{P}(6)-\mathrm{C}(69)$

$\mathrm{P}(5)-\operatorname{Ir}(3)-\mathrm{P}(6)-\mathrm{C}(69)$

$\mathrm{N}(5)-\operatorname{Ir}(3)-\mathrm{P}(6)-\mathrm{C}(65)$

$\mathrm{C}(49)-\operatorname{Ir}(3)-\mathrm{P}(6)-\mathrm{C}(65)$

$\mathrm{P}(5)-\operatorname{Ir}(3)-\mathrm{P}(6)-\mathrm{C}(65)$

$\mathrm{N}(5)-\operatorname{Ir}(3)-\mathrm{C}(49)-\mathrm{C}(50)$

$\mathrm{P}(6)-\operatorname{Ir}(3)-\mathrm{C}(49)-\mathrm{C}(50)$

$\mathrm{P}(5)-\operatorname{Ir}(3)-\mathrm{C}(49)-\mathrm{C}(50)$

$\mathrm{N}(5)-\operatorname{Ir}(3)-\mathrm{C}(49)-\mathrm{C}(54)$

$\mathrm{P}(6)-\operatorname{Ir}(3)-\mathrm{C}(49)-\mathrm{C}(54)$

$\mathrm{P}(5)-\operatorname{Ir}(3)-\mathrm{C}(49)-\mathrm{C}(54)$

$\mathrm{C}(54)-\mathrm{C}(49)-\mathrm{C}(50)-\mathrm{C}(51)$

$\operatorname{Ir}(3)-\mathrm{C}(49)-\mathrm{C}(50)-\mathrm{C}(51)$

$\mathrm{C}(54)-\mathrm{C}(49)-\mathrm{C}(50)-\mathrm{C}(55)$

$\operatorname{Ir}(3)-\mathrm{C}(49)-\mathrm{C}(50)-\mathrm{C}(55)$

$\mathrm{C}(49)-\mathrm{C}(50)-\mathrm{C}(51)-\mathrm{C}(52)$

$\mathrm{C}(55)-\mathrm{C}(50)-\mathrm{C}(51)-\mathrm{C}(52)$

$\mathrm{C}(50)-\mathrm{C}(51)-\mathrm{C}(52)-\mathrm{C}(53)$

$\mathrm{C}(51)-\mathrm{C}(52)-\mathrm{C}(53)-\mathrm{C}(54)$

$\mathrm{C}(52)-\mathrm{C}(53)-\mathrm{C}(54)-\mathrm{C}(49)$

$\mathrm{C}(52)-\mathrm{C}(53)-\mathrm{C}(54)-\mathrm{C}(56)$

$\mathrm{C}(50)-\mathrm{C}(49)-\mathrm{C}(54)-\mathrm{C}(53)$

$\operatorname{Ir}(3)-\mathrm{C}(49)-\mathrm{C}(54)-\mathrm{C}(53)$

$\mathrm{C}(50)-\mathrm{C}(49)-\mathrm{C}(54)-\mathrm{C}(56)$

$\operatorname{Ir}(3)-\mathrm{C}(49)-\mathrm{C}(54)-\mathrm{C}(56)$

$\mathrm{C}(51)-\mathrm{C}(50)-\mathrm{C}(55)-\mathrm{P}(5)$

$\mathrm{C}(49)-\mathrm{C}(50)-\mathrm{C}(55)-\mathrm{P}(5)$

$\mathrm{C}(61)-\mathrm{P}(5)-\mathrm{C}(55)-\mathrm{C}(50)$

$\mathrm{C}(57)-\mathrm{P}(5)-\mathrm{C}(55)-\mathrm{C}(50)$

$\operatorname{Ir}(3)-\mathrm{P}(5)-\mathrm{C}(55)-\mathrm{C}(50)$

$\mathrm{C}(53)-\mathrm{C}(54)-\mathrm{C}(56)-\mathrm{P}(6)$

$\mathrm{C}(49)-\mathrm{C}(54)-\mathrm{C}(56)-\mathrm{P}(6)$

$\mathrm{C}(69)-\mathrm{P}(6)-\mathrm{C}(56)-\mathrm{C}(54)$

$\mathrm{C}(65)-\mathrm{P}(6)-\mathrm{C}(56)-\mathrm{C}(54)$

$\operatorname{Ir}(3)-\mathrm{P}(6)-\mathrm{C}(56)-\mathrm{C}(54)$

$\mathrm{C}(55)-\mathrm{P}(5)-\mathrm{C}(57)-\mathrm{C}(58)$

$\mathrm{C}(61)-\mathrm{P}(5)-\mathrm{C}(57)-\mathrm{C}(58)$

$\operatorname{Ir}(3)-\mathrm{P}(5)-\mathrm{C}(57)-\mathrm{C}(58)$

$\mathrm{C}(55)-\mathrm{P}(5)-\mathrm{C}(57)-\mathrm{C}(59)$

$\mathrm{C}(61)-\mathrm{P}(5)-\mathrm{C}(57)-\mathrm{C}(59)$

$\operatorname{Ir}(3)-\mathrm{P}(5)-\mathrm{C}(57)-\mathrm{C}(59)$

$\mathrm{C}(55)-\mathrm{P}(5)-\mathrm{C}(57)-\mathrm{C}(60)$

$\mathrm{C}(61)-\mathrm{P}(5)-\mathrm{C}(57)-\mathrm{C}(60)$

$\operatorname{Ir}(3)-\mathrm{P}(5)-\mathrm{C}(57)-\mathrm{C}(60)$

$\mathrm{C}(55)-\mathrm{P}(5)-\mathrm{C}(61)-\mathrm{C}(64)$

$\mathrm{C}(57)-\mathrm{P}(5)-\mathrm{C}(61)-\mathrm{C}(64)$

$\operatorname{Ir}(3)-\mathrm{P}(5)-\mathrm{C}(61)-\mathrm{C}(64)$

$\mathrm{C}(55)-\mathrm{P}(5)-\mathrm{C}(61)-\mathrm{C}(63)$

$\mathrm{C}(57)-\mathrm{P}(5)-\mathrm{C}(61)-\mathrm{C}(63)$

$\operatorname{Ir}(3)-\mathrm{P}(5)-\mathrm{C}(61)-\mathrm{C}(63)$

$\mathrm{C}(55)-\mathrm{P}(5)-\mathrm{C}(61)-\mathrm{C}(62)$

$\mathrm{C}(57)-\mathrm{P}(5)-\mathrm{C}(61)-\mathrm{C}(62)$

$\operatorname{Ir}(3)-\mathrm{P}(5)-\mathrm{C}(61)-\mathrm{C}(62)$

$\mathrm{C}(56)-\mathrm{P}(6)-\mathrm{C}(65)-\mathrm{C}(68)$

$\mathrm{C}(69)-\mathrm{P}(6)-\mathrm{C}(65)-\mathrm{C}(68)$

$\operatorname{Ir}(3)-\mathrm{P}(6)-\mathrm{C}(65)-\mathrm{C}(68)$

$\mathrm{C}(56)-\mathrm{P}(6)-\mathrm{C}(65)-\mathrm{C}(66)$

$\mathrm{C}(69)-\mathrm{P}(6)-\mathrm{C}(65)-\mathrm{C}(66)$

$\operatorname{Ir}(3)-\mathrm{P}(6)-\mathrm{C}(65)-\mathrm{C}(66)$

$\mathrm{C}(56)-\mathrm{P}(6)-\mathrm{C}(65)-\mathrm{C}(67)$

$\mathrm{C}(69)-\mathrm{P}(6)-\mathrm{C}(65)-\mathrm{C}(67)$

$\operatorname{Ir}(3)-\mathrm{P}(6)-\mathrm{C}(65)-\mathrm{C}(67)$

\footnotetext{
12.62(10)

$80.28(9)$

$-100.55(8)$

$-100.70(9)$

$-52.60(9)$

126.56(9)

126.42(9)

$-110(5)$

174.02(15)

$-6.01(14)$

$68(5)$

$-7.25(14)$

172.72(15) $-1.5(3)$

177.27(14)

176.33(16)

$-4.9(2)$

2.9(3)

$-175.00(17)$

$-1.4(3)$

$-1.2(3)$

$2.5(3)$

$-174.19(17)$

$-1.1(3)$

$-179.94(14)$

175.55(16)

$-3.2(2)$

$-166.52(14)$

$15.6(2)$

$-142.04(13)$

101.08(14)

$-17.54(14)$

$-168.56(14)$

$14.6(2)$

101.64(14)

$-141.91(13)$

$-17.55(14)$

70.73(17)

$-40.45(18)$

$-176.68(13)$

$-50.10(16)$

$-161.27(14)$

$62.50(15)$

$-165.24(13)$

$83.58(15)$

$-52.65(14)$

$-171.23(15)$

$-59.09(17)$

$74.32(15)$

$72.05(15)$

$-175.82(14)$

$-42.40(15)$

$-47.34(16)$

$64.80(16)$

$-161.79(12)$

$-170.03(14)$

$-58.20(16)$

$75.57(15)$

$72.85(14)$

$-175.31(13)$

$-41.54(15)$

$-45.47(16)$

66.36(16)

$-159.87(12)$
} 
$\mathrm{C}(56)-\mathrm{P}(6)-\mathrm{C}(69)-\mathrm{C}(71)$ $\mathrm{C}(65)-\mathrm{P}(6)-\mathrm{C}(69)-\mathrm{C}(71)$ $\operatorname{Ir}(3)-\mathrm{P}(6)-\mathrm{C}(69)-\mathrm{C}(71)$ $\mathrm{C}(56)-\mathrm{P}(6)-\mathrm{C}(69)-\mathrm{C}(72)$ $\mathrm{C}(65)-\mathrm{P}(6)-\mathrm{C}(69)-\mathrm{C}(72)$ $\operatorname{Ir}(3)-\mathrm{P}(6)-\mathrm{C}(69)-\mathrm{C}(72)$ $\mathrm{C}(56)-\mathrm{P}(6)-\mathrm{C}(69)-\mathrm{C}(70)$ $\mathrm{C}(65)-\mathrm{P}(6)-\mathrm{C}(69)-\mathrm{C}(70)$ $\operatorname{Ir}(3)-\mathrm{P}(6)-\mathrm{C}(69)-\mathrm{C}(70)$ $\mathrm{C}(73)-\operatorname{Ir}(4)-\mathrm{N}(7)-\mathrm{N}(8)$ $\mathrm{P}(8)-\operatorname{Ir}(4)-\mathrm{N}(7)-\mathrm{N}(8)$ $\mathrm{P}(7)-\operatorname{Ir}(4)-\mathrm{N}(7)-\mathrm{N}(8)$ $\mathrm{N}(7)-\operatorname{Ir}(4)-\mathrm{P}(7)-\mathrm{C}(79)$ $\mathrm{C}(73)-\operatorname{Ir}(4)-\mathrm{P}(7)-\mathrm{C}(79)$ $\mathrm{P}(8)-\operatorname{Ir}(4)-\mathrm{P}(7)-\mathrm{C}(79)$ $\mathrm{N}(7)-\operatorname{Ir}(4)-\mathrm{P}(7)-\mathrm{C}(85)$ $\mathrm{C}(73)-\operatorname{Ir}(4)-\mathrm{P}(7)-\mathrm{C}(85)$ $\mathrm{P}(8)-\operatorname{Ir}(4)-\mathrm{P}(7)-\mathrm{C}(85)$ $\mathrm{N}(7)-\operatorname{Ir}(4)-\mathrm{P}(7)-\mathrm{C}(81)$ $\mathrm{C}(73)-\operatorname{Ir}(4)-\mathrm{P}(7)-\mathrm{C}(81)$ $\mathrm{P}(8)-\operatorname{Ir}(4)-\mathrm{P}(7)-\mathrm{C}(81)$ $\mathrm{N}(7)-\operatorname{Ir}(4)-\mathrm{P}(8)-\mathrm{C}(80)$ $\mathrm{C}(73)-\operatorname{Ir}(4)-\mathrm{P}(8)-\mathrm{C}(80)$ $\mathrm{P}(7)-\operatorname{Ir}(4)-\mathrm{P}(8)-\mathrm{C}(80)$ $\mathrm{N}(7)-\operatorname{Ir}(4)-\mathrm{P}(8)-\mathrm{C}(93)$ $\mathrm{C}(73)-\operatorname{Ir}(4)-\mathrm{P}(8)-\mathrm{C}(93)$ $\mathrm{P}(7)-\operatorname{Ir}(4)-\mathrm{P}(8)-\mathrm{C}(93)$ $\mathrm{N}(7)-\operatorname{Ir}(4)-\mathrm{P}(8)-\mathrm{C}(89)$ $\mathrm{C}(73)-\operatorname{Ir}(4)-\mathrm{P}(8)-\mathrm{C}(89)$ $\mathrm{P}(7)-\operatorname{Ir}(4)-\mathrm{P}(8)-\mathrm{C}(89)$ $\mathrm{N}(7)-\operatorname{Ir}(4)-\mathrm{C}(73)-\mathrm{C}(74)$ $\mathrm{P}(8)-\operatorname{Ir}(4)-\mathrm{C}(73)-\mathrm{C}(74)$ $\mathrm{P}(7)-\operatorname{Ir}(4)-\mathrm{C}(73)-\mathrm{C}(74)$ $\mathrm{N}(7)-\operatorname{Ir}(4)-\mathrm{C}(73)-\mathrm{C}(78)$ $\mathrm{P}(8)-\operatorname{Ir}(4)-\mathrm{C}(73)-\mathrm{C}(78)$ $\mathrm{P}(7)-\operatorname{Ir}(4)-\mathrm{C}(73)-\mathrm{C}(78)$ $\mathrm{C}(78)-\mathrm{C}(73)-\mathrm{C}(74)-\mathrm{C}(75)$ $\operatorname{Ir}(4)-\mathrm{C}(73)-\mathrm{C}(74)-\mathrm{C}(75)$ $\mathrm{C}(78)-\mathrm{C}(73)-\mathrm{C}(74)-\mathrm{C}(79)$ $\operatorname{Ir}(4)-\mathrm{C}(73)-\mathrm{C}(74)-\mathrm{C}(79)$ $\mathrm{C}(73)-\mathrm{C}(74)-\mathrm{C}(75)-\mathrm{C}(76)$ $\mathrm{C}(79)-\mathrm{C}(74)-\mathrm{C}(75)-\mathrm{C}(76)$ $\mathrm{C}(74)-\mathrm{C}(75)-\mathrm{C}(76)-\mathrm{C}(77)$ $\mathrm{C}(75)-\mathrm{C}(76)-\mathrm{C}(77)-\mathrm{C}(78)$ $\mathrm{C}(76)-\mathrm{C}(77)-\mathrm{C}(78)-\mathrm{C}(73)$ $\mathrm{C}(76)-\mathrm{C}(77)-\mathrm{C}(78)-\mathrm{C}(80)$ $\mathrm{C}(74)-\mathrm{C}(73)-\mathrm{C}(78)-\mathrm{C}(77)$ $\operatorname{Ir}(4)-\mathrm{C}(73)-\mathrm{C}(78)-\mathrm{C}(77)$

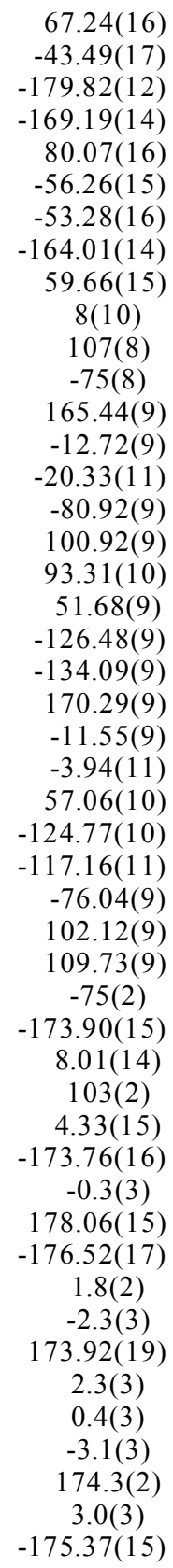

$\mathrm{C}(74)-\mathrm{C}(73)-\mathrm{C}(78)-\mathrm{C}(80)$ $\operatorname{Ir}(4)-\mathrm{C}(73)-\mathrm{C}(78)-\mathrm{C}(80)$ $\mathrm{C}(75)-\mathrm{C}(74)-\mathrm{C}(79)-\mathrm{P}(7)$ C(73)-C(74)-C(79)-P(7) $\mathrm{C}(85)-\mathrm{P}(7)-\mathrm{C}(79)-\mathrm{C}(74)$ $\mathrm{C}(81)-\mathrm{P}(7)-\mathrm{C}(79)-\mathrm{C}(74)$ $\operatorname{Ir}(4)-\mathrm{P}(7)-\mathrm{C}(79)-\mathrm{C}(74)$ $\mathrm{C}(77)-\mathrm{C}(78)-\mathrm{C}(80)-\mathrm{P}(8)$ $\mathrm{C}(73)-\mathrm{C}(78)-\mathrm{C}(80)-\mathrm{P}(8)$ $\mathrm{C}(93)-\mathrm{P}(8)-\mathrm{C}(80)-\mathrm{C}(78)$ $\mathrm{C}(89)-\mathrm{P}(8)-\mathrm{C}(80)-\mathrm{C}(78)$ $\operatorname{Ir}(4)-\mathrm{P}(8)-\mathrm{C}(80)-\mathrm{C}(78)$ $\mathrm{C}(79)-\mathrm{P}(7)-\mathrm{C}(81)-\mathrm{C}(83)$ C(85)-P(7)-C(81)-C(83) $\operatorname{Ir}(4)-\mathrm{P}(7)-\mathrm{C}(81)-\mathrm{C}(83)$ $\mathrm{C}(79)-\mathrm{P}(7)-\mathrm{C}(81)-\mathrm{C}(82)$ $\mathrm{C}(85)-\mathrm{P}(7)-\mathrm{C}(81)-\mathrm{C}(82)$ $\operatorname{Ir}(4)-\mathrm{P}(7)-\mathrm{C}(81)-\mathrm{C}(82)$ $\mathrm{C}(79)-\mathrm{P}(7)-\mathrm{C}(81)-\mathrm{C}(84)$ $\mathrm{C}(85)-\mathrm{P}(7)-\mathrm{C}(81)-\mathrm{C}(84)$ $\operatorname{Ir}(4)-\mathrm{P}(7)-\mathrm{C}(81)-\mathrm{C}(84)$ $\mathrm{C}(79)-\mathrm{P}(7)-\mathrm{C}(85)-\mathrm{C}(86)$ $\mathrm{C}(81)-\mathrm{P}(7)-\mathrm{C}(85)-\mathrm{C}(86)$ $\operatorname{Ir}(4)-\mathrm{P}(7)-\mathrm{C}(85)-\mathrm{C}(86)$ $\mathrm{C}(79)-\mathrm{P}(7)-\mathrm{C}(85)-\mathrm{C}(88)$ $\mathrm{C}(81)-\mathrm{P}(7)-\mathrm{C}(85)-\mathrm{C}(88)$ $\operatorname{Ir}(4)-\mathrm{P}(7)-\mathrm{C}(85)-\mathrm{C}(88)$ $\mathrm{C}(79)-\mathrm{P}(7)-\mathrm{C}(85)-\mathrm{C}(87)$ $\mathrm{C}(81)-\mathrm{P}(7)-\mathrm{C}(85)-\mathrm{C}(87)$ $\operatorname{Ir}(4)-\mathrm{P}(7)-\mathrm{C}(85)-\mathrm{C}(87)$ $\mathrm{C}(80)-\mathrm{P}(8)-\mathrm{C}(89)-\mathrm{C}(92)$ $\mathrm{C}(93)-\mathrm{P}(8)-\mathrm{C}(89)-\mathrm{C}(92)$ $\operatorname{Ir}(4)-\mathrm{P}(8)-\mathrm{C}(89)-\mathrm{C}(92)$ C(80)-P(8)-C(89)-C(91) $\mathrm{C}(93)-\mathrm{P}(8)-\mathrm{C}(89)-\mathrm{C}(91)$ $\operatorname{Ir}(4)-\mathrm{P}(8)-\mathrm{C}(89)-\mathrm{C}(91)$ $\mathrm{C}(80)-\mathrm{P}(8)-\mathrm{C}(89)-\mathrm{C}(90)$ C(93)-P(8)-C(89)-C(90) $\operatorname{Ir}(4)-\mathrm{P}(8)-\mathrm{C}(89)-\mathrm{C}(90)$ $\mathrm{C}(80)-\mathrm{P}(8)-\mathrm{C}(93)-\mathrm{C}(95)$ $\mathrm{C}(89)-\mathrm{P}(8)-\mathrm{C}(93)-\mathrm{C}(95)$ $\operatorname{Ir}(4)-\mathrm{P}(8)-\mathrm{C}(93)-\mathrm{C}(95)$ $\mathrm{C}(80)-\mathrm{P}(8)-\mathrm{C}(93)-\mathrm{C}(94)$ $\mathrm{C}(89)-\mathrm{P}(8)-\mathrm{C}(93)-\mathrm{C}(94)$ $\operatorname{Ir}(4)-\mathrm{P}(8)-\mathrm{C}(93)-\mathrm{C}(94)$ $\mathrm{C}(80)-\mathrm{P}(8)-\mathrm{C}(93)-\mathrm{C}(96)$ C(89)-P(8)-C(93)-C(96) $\operatorname{Ir}(4)-\mathrm{P}(8)-\mathrm{C}(93)-\mathrm{C}(96)$
$-174.42(17)$ $7.2(3)$

170.31(15) $-13.3(2)$

$-101.56(14)$

$141.48(14)$

16.91(14)

165.47(16) $-17.1(2)$

141.29(15)

$-101.93(15)$

$17.80(16)$

$-73.00(14)$

$174.33(13)$

41.31(15)

$45.77(16)$

$-66.91(16)$

$160.07(12)$

$169.80(15)$

$57.12(17)$

$-75.90(15)$

$-69.34(16)$

41.78(17)

177.91(13)

167.07(14)

$-81.81(15)$

54.32(15)

$51.08(16)$

$162.21(14)$

$-61.66(15)$

$165.84(14)$

$-83.33(16)$

52.74(15)

$-70.39(17)$

40.44(18)

176.51(13)

$50.19(16)$

$161.02(15)$

$-62.91(15)$

$170.27(17)$

$58.16(19)$

$-75.91(17)$

46.45(19)

$-65.66(19)$

$160.27(14)$

$-72.77(19)$

175.12(17)

41.0(2) 
Figure S-2 : ORTEP Diagram of complex 2

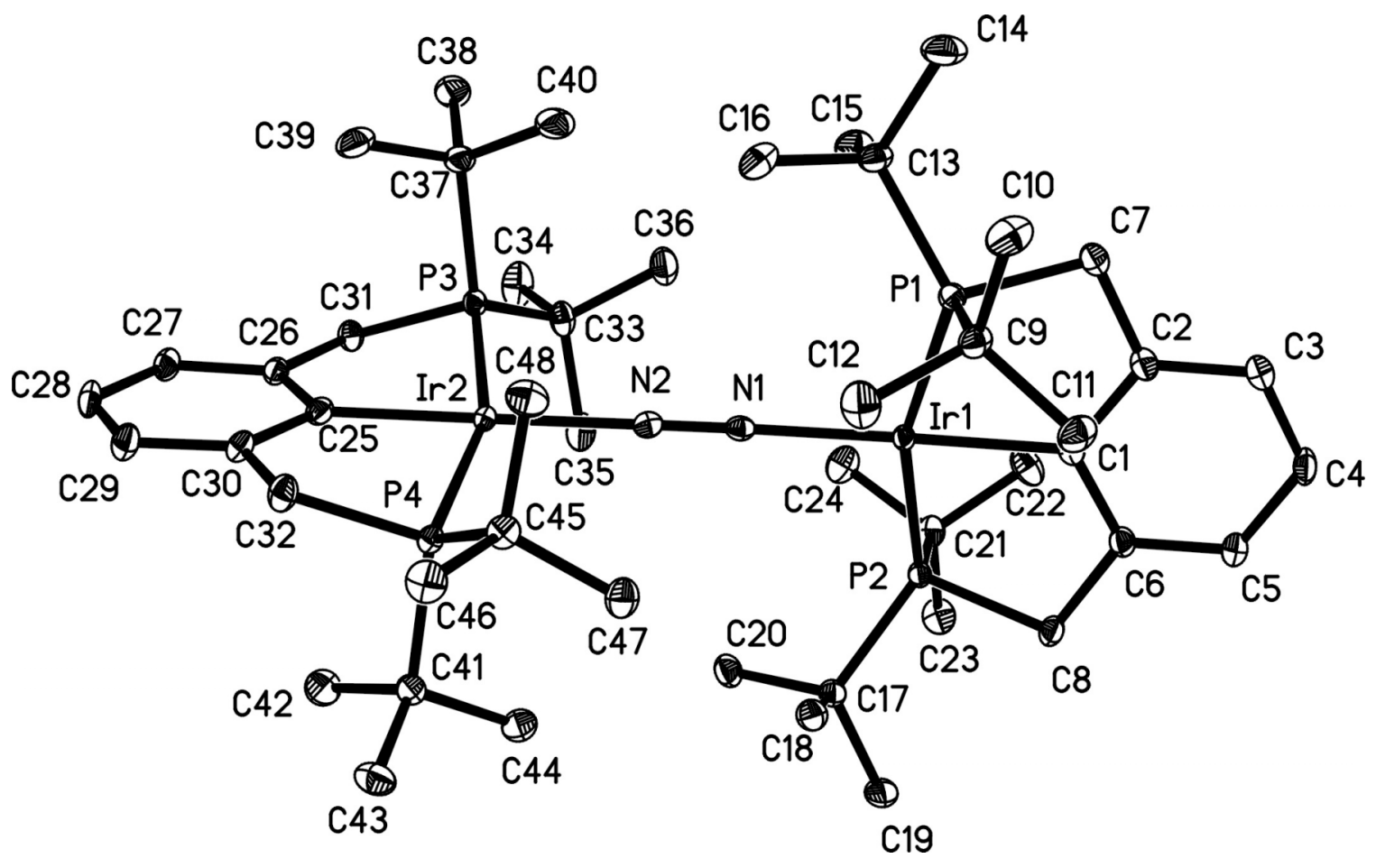


Table S-5. Crystal data and structure refinement for complex 2.

Identification code

Empirical formula

Formula weight

Temperature

Wavelength

Crystal system

Space group

Unit cell dimensions

Volume

Z

Density (calculated)

Absorption coefficient

$\mathrm{F}(000)$

Crystal size

Theta range for data collection

Index ranges

Reflections collected

Independent reflections

Completeness to theta $=30.61^{\circ}$

Absorption correction

Max. and min. transmission

Refinement method

Data / restraints / parameters

Goodness-of-fit on $\mathrm{F}^{2}$

Final $\mathrm{R}$ indices [I $>2 \operatorname{sigma}(\mathrm{I})]$

$\mathrm{R}$ indices (all data)

Largest diff. peak and hole irhbzome

C51 H93 Ir2 N2 P4

1242.55

$100(2) \mathrm{K}$

$0.71073 \AA$

Triclinic

P-1

$\begin{array}{ll}\mathrm{a}=14.2815(8) \AA & \alpha=70.5540(10)^{\circ} . \\ \mathrm{b}=14.4823(8) \AA & \beta=89.0900(10)^{\circ} . \\ \mathrm{c}=14.7312(8) \AA & \gamma=69.7850(10)^{\circ} .\end{array}$

2679.1(3) $\AA^{3}$

2

$1.540 \mathrm{Mg} / \mathrm{m}^{3}$

$5.115 \mathrm{~mm}^{-1}$

1254

$0.42 \times 0.33 \times 0.22 \mathrm{~mm}^{3}$

2.00 to $30.61^{\circ}$.

$-20<=\mathrm{h}<=20,-20<=\mathrm{k}<=20,-21<=1<=21$

36737

$16290[\mathrm{R}(\mathrm{int})=0.0193]$

$98.7 \%$

Semi-empirical from equivalents

0.9999 and 0.6962

Full-matrix least-squares on $\mathrm{F}^{2}$

16290 / 0 / 557

1.007

$\mathrm{R} 1=0.0190, \mathrm{wR} 2=0.0459$

$\mathrm{R} 1=0.0213, \mathrm{wR} 2=0.0468$

1.734 and -1.030 e. $\AA^{-3}$ 
Table S-6. Atomic coordinates ( $\left.\times 10^{4}\right)$ and equivalent isotropic displacement parameters $\left(\AA^{2} \times 10^{3}\right)$ for complex 2. $\mathrm{U}(\mathrm{eq})$ is defined as one third of the trace of the orthogonalized $\mathrm{U}^{\mathrm{ij}}$ tensor.

\begin{tabular}{|c|c|c|c|c|}
\hline & $\mathrm{x}$ & $\mathrm{y}$ & $\mathrm{z}$ & $\mathrm{U}(\mathrm{eq})$ \\
\hline$\overline{\operatorname{Ir}}(1)$ & $7658(1)$ & $2841(1)$ & $4374(1)$ & $9(1)$ \\
\hline $\operatorname{Ir}(2)$ & $5097(1)$ & $2332(1)$ & $2338(1)$ & $9(1)$ \\
\hline $\mathrm{N}(1)$ & $6667(1)$ & $2648(1)$ & $3565(1)$ & $11(1)$ \\
\hline$N(2)$ & $6100(1)$ & $2538(1)$ & $3121(1)$ & $11(1)$ \\
\hline $\mathrm{P}(1)$ & $8208(1)$ & $3972(1)$ & $3190(1)$ & $11(1)$ \\
\hline $\mathrm{P}(2)$ & $7468(1)$ & $1801(1)$ & $5879(1)$ & $10(1)$ \\
\hline $\mathrm{P}(3)$ & $6144(1)$ & 886(1) & $2026(1)$ & $11(1)$ \\
\hline $\mathrm{P}(4)$ & $3675(1)$ & $3711(1)$ & $2327(1)$ & $10(1)$ \\
\hline$C(1)$ & $8657(1)$ & $3041(1)$ & $5211(1)$ & $13(1)$ \\
\hline $\mathrm{C}(2)$ & 9403(1) & $3444(2)$ & $4818(1)$ & $14(1)$ \\
\hline$C(3)$ & $10080(2)$ & $3580(2)$ & $5391(1)$ & $17(1)$ \\
\hline$C(4)$ & $10050(2)$ & $3302(2)$ & $6388(1)$ & $17(1)$ \\
\hline$C(5)$ & $9327(2)$ & $2902(2)$ & $6803(1)$ & $16(1)$ \\
\hline$C(6)$ & $8643(1)$ & $2782(1)$ & $6228(1)$ & $13(1)$ \\
\hline$C(7)$ & $9446(1)$ & $3752(2)$ & $3741(1)$ & $16(1)$ \\
\hline $\mathrm{C}(8)$ & $7861(2)$ & $2359(2)$ & $6689(1)$ & $14(1)$ \\
\hline C(9) & $7483(2)$ & $5412(2)$ & $2950(1)$ & $15(1)$ \\
\hline$C(10)$ & $7836(2)$ & $6165(2)$ & $2143(2)$ & $22(1)$ \\
\hline $\mathrm{C}(11)$ & $7586(2)$ & $5625(2)$ & $3892(1)$ & $19(1)$ \\
\hline $\mathrm{C}(12)$ & $6372(2)$ & $5634(2)$ & $2710(2)$ & $23(1)$ \\
\hline $\mathrm{C}(13)$ & $8505(2)$ & $3733(2)$ & $2021(1)$ & $15(1)$ \\
\hline $\mathrm{C}(14)$ & $9270(2)$ & $4190(2)$ & $1492(2)$ & $24(1)$ \\
\hline$C(15)$ & $8972(2)$ & $2532(2)$ & $2309(2)$ & $20(1)$ \\
\hline $\mathrm{C}(16)$ & $7544(2)$ & $4133(2)$ & $1334(1)$ & $20(1)$ \\
\hline $\mathrm{C}(17)$ & $6167(1)$ & 1899(2) & $6197(1)$ & $13(1)$ \\
\hline $\mathrm{C}(18)$ & $5499(1)$ & $3052(2)$ & $5665(1)$ & $16(1)$ \\
\hline $\mathrm{C}(19)$ & $6056(2)$ & $1645(2)$ & $7288(1)$ & $18(1)$ \\
\hline $\mathrm{C}(20)$ & $5798(2)$ & $1202(2)$ & $5823(1)$ & $17(1)$ \\
\hline $\mathrm{C}(21)$ & $8364(1)$ & 392(1) & $6317(1)$ & $14(1)$ \\
\hline $\mathrm{C}(22)$ & $9434(2)$ & $410(2)$ & $6365(2)$ & $21(1)$ \\
\hline $\mathrm{C}(23)$ & $8205(2)$ & $-285(2)$ & $7314(1)$ & $20(1)$ \\
\hline $\mathrm{C}(24)$ & $8281(2)$ & $-96(2)$ & $5560(2)$ & $20(1)$ \\
\hline $\mathrm{C}(25)$ & $4084(1)$ & $2114(1)$ & $1537(1)$ & $12(1)$ \\
\hline$C(26)$ & $4282(1)$ & $1155(1)$ & $1373(1)$ & $12(1)$ \\
\hline$C(27)$ & $3601(2)$ & $1008(2)$ & $814(1)$ & $15(1)$ \\
\hline $\mathrm{C}(28)$ & $2678(2)$ & $1807(2)$ & $408(1)$ & $17(1)$ \\
\hline $\mathrm{C}(29)$ & $2454(2)$ & $2750(2)$ & $566(1)$ & $17(1)$ \\
\hline$C(30)$ & $3144(1)$ & $2906(2)$ & $1102(1)$ & $14(1)$ \\
\hline $\mathrm{C}(31)$ & $5273(1)$ & 281(1) & $1812(1)$ & $13(1)$ \\
\hline$C(32)$ & $2898(2)$ & $3945(1)$ & $1238(1)$ & $15(1)$ \\
\hline$C(33)$ & $7086(1)$ & $-233(1)$ & $3011(1)$ & $15(1)$ \\
\hline $\mathrm{C}(34)$ & $7339(2)$ & $-1314(2)$ & $2905(2)$ & $25(1)$ \\
\hline$C(35)$ & $6584(2)$ & $-264(2)$ & $3944(1)$ & $19(1)$ \\
\hline $\mathrm{C}(36)$ & $8062(2)$ & $-36(2)$ & $3109(2)$ & $22(1)$ \\
\hline $\mathrm{C}(37)$ & $6713(2)$ & $1193(2)$ & 853(1) & $16(1)$ \\
\hline $\mathrm{C}(38)$ & $7399(2)$ & $224(2)$ & $647(2)$ & $24(1)$ \\
\hline $\mathrm{C}(39)$ & $5855(2)$ & $1818(2)$ & 19(1) & $21(1)$ \\
\hline $\mathrm{C}(40)$ & $7297(2)$ & $1905(2)$ & $865(2)$ & $25(1)$ \\
\hline$C(41)$ & $2862(1)$ & $3412(2)$ & $3324(1)$ & $14(1)$ \\
\hline$C(42)$ & $2511(2)$ & $2553(2)$ & $3231(2)$ & $20(1)$ \\
\hline$C(43)$ & $1937(2)$ & $4350(2)$ & $3315(2)$ & $20(1)$ \\
\hline $\mathrm{C}(44)$ & $3513(2)$ & $2956(2)$ & $4297(1)$ & $19(1)$ \\
\hline$C(45)$ & $3771(1)$ & $5027(1)$ & $2096(1)$ & $14(1)$ \\
\hline$C(46)$ & $2782(2)$ & $5970(2)$ & $1662(2)$ & $19(1)$ \\
\hline $\mathrm{C}(47)$ & $4170(2)$ & $5080(2)$ & $3030(1)$ & $17(1)$ \\
\hline $\mathrm{C}(48)$ & $4534(2)$ & $5119(2)$ & $1354(2)$ & $19(1)$ \\
\hline C(49) & $9882(2)$ & $590(3)$ & $9701(2)$ & $44(1)$ \\
\hline $\mathrm{C}(50)$ & $10199(2)$ & $1157(3)$ & $10252(2)$ & $47(1)$ \\
\hline $\mathrm{C}(51)$ & $9988(2)$ & $2298(3)$ & $9655(2)$ & $46(1)$ \\
\hline
\end{tabular}


Table S-7. Bond lengths $[\AA]$ and angles $\left[{ }^{\circ}\right]$ for complex 2.

\begin{tabular}{|c|c|c|c|}
\hline $\operatorname{Ir}(1)-\mathrm{N}(1)$ & $2.0121(16)$ & $\overline{\mathrm{C}}(\overline{17})-\overline{\mathrm{C}}(19)$ & $1.543(3)$ \\
\hline $\operatorname{Ir}(1)-C(1)$ & $2.0534(18)$ & $\mathrm{C}(18)-\mathrm{H}(18 \mathrm{~A})$ & 0.9800 \\
\hline $\operatorname{Ir}(1)-\mathrm{P}(1)$ & $2.2989(5)$ & $\mathrm{C}(18)-\mathrm{H}(18 \mathrm{~B})$ & 0.9800 \\
\hline $\operatorname{Ir}(1)-P(2)$ & $2.3028(5)$ & $\mathrm{C}(18)-\mathrm{H}(18 \mathrm{C})$ & 0.9800 \\
\hline $\operatorname{Ir}(2)-\mathrm{N}(2)$ & $2.0115(16)$ & $\mathrm{C}(19)-\mathrm{H}(19 \mathrm{~A})$ & 0.9800 \\
\hline $\operatorname{Ir}(2)-C(25)$ & $2.0511(18)$ & $\mathrm{C}(19)-\mathrm{H}(19 \mathrm{~B})$ & 0.9800 \\
\hline $\operatorname{Ir}(2)-P(3)$ & $2.2985(5)$ & $\mathrm{C}(19)-\mathrm{H}(19 \mathrm{C})$ & 0.9800 \\
\hline $\operatorname{Ir}(2)-P(4)$ & $2.3001(5)$ & $\mathrm{C}(20)-\mathrm{H}(20 \mathrm{~A})$ & 0.9800 \\
\hline $\mathrm{N}(1)-\mathrm{N}(2)$ & $1.134(2)$ & $\mathrm{C}(20)-\mathrm{H}(20 \mathrm{~B})$ & 0.9800 \\
\hline $\mathrm{P}(1)-\mathrm{C}(7)$ & $1.8364(19)$ & $\mathrm{C}(20)-\mathrm{H}(20 \mathrm{C})$ & 0.9800 \\
\hline $\mathrm{P}(1)-\mathrm{C}(13)$ & $1.8804(19)$ & $\mathrm{C}(21)-\mathrm{C}(24)$ & $1.530(3)$ \\
\hline $\mathrm{P}(1)-\mathrm{C}(9)$ & $1.8902(19)$ & $\mathrm{C}(21)-\mathrm{C}(23)$ & $1.534(3)$ \\
\hline $\mathrm{P}(2)-\mathrm{C}(8)$ & $1.8352(18)$ & $\mathrm{C}(21)-\mathrm{C}(22)$ & $1.541(3)$ \\
\hline $\mathrm{P}(2)-\mathrm{C}(17)$ & $1.8768(19)$ & $\mathrm{C}(22)-\mathrm{H}(22 \mathrm{~A})$ & 0.9800 \\
\hline$P(2)-C(21)$ & $1.8873(19)$ & $\mathrm{C}(22)-\mathrm{H}(22 \mathrm{~B})$ & 0.9800 \\
\hline $\mathrm{P}(3)-\mathrm{C}(31)$ & $1.8345(18)$ & $\mathrm{C}(22)-\mathrm{H}(22 \mathrm{C})$ & 0.9800 \\
\hline $\mathrm{P}(3)-\mathrm{C}(33)$ & $1.880(2)$ & $\mathrm{C}(23)-\mathrm{H}(23 \mathrm{~A})$ & 0.9800 \\
\hline $\mathrm{P}(3)-\mathrm{C}(37)$ & $1.8818(19)$ & $\mathrm{C}(23)-\mathrm{H}(23 \mathrm{~B})$ & 0.9800 \\
\hline $\mathrm{P}(4)-\mathrm{C}(32)$ & $1.8309(19)$ & $\mathrm{C}(23)-\mathrm{H}(23 \mathrm{C})$ & 0.9800 \\
\hline$P(4)-C(45)$ & $1.8746(19)$ & $\mathrm{C}(24)-\mathrm{H}(24 \mathrm{~A})$ & 0.9800 \\
\hline $\mathrm{P}(4)-\mathrm{C}(41)$ & $1.8878(19)$ & $\mathrm{C}(24)-\mathrm{H}(24 \mathrm{~B})$ & 0.9800 \\
\hline $\mathrm{C}(1)-\mathrm{C}(2)$ & $1.414(3)$ & $\mathrm{C}(24)-\mathrm{H}(24 \mathrm{C})$ & 0.9800 \\
\hline $\mathrm{C}(1)-\mathrm{C}(6)$ & $1.420(3)$ & $\mathrm{C}(25)-\mathrm{C}(30)$ & $1.415(3)$ \\
\hline$C(2)-C(3)$ & $1.398(3)$ & $\mathrm{C}(25)-\mathrm{C}(26)$ & $1.420(2)$ \\
\hline $\mathrm{C}(2)-\mathrm{C}(7)$ & $1.505(3)$ & $\mathrm{C}(26)-\mathrm{C}(27)$ & $1.398(2)$ \\
\hline$C(3)-C(4)$ & $1.393(3)$ & $C(26)-C(31)$ & $1.506(3)$ \\
\hline $\mathrm{C}(3)-\mathrm{H}(3)$ & 0.9500 & $\mathrm{C}(27)-\mathrm{C}(28)$ & $1.394(3)$ \\
\hline$C(4)-C(5)$ & $1.390(3)$ & $\mathrm{C}(27)-\mathrm{H}(27)$ & 0.9500 \\
\hline $\mathrm{C}(4)-\mathrm{H}(4)$ & 0.9500 & $\mathrm{C}(28)-\mathrm{C}(29)$ & $1.389(3)$ \\
\hline $\mathrm{C}(5)-\mathrm{C}(6)$ & $1.394(2)$ & $\mathrm{C}(28)-\mathrm{H}(28)$ & 0.9500 \\
\hline $\mathrm{C}(5)-\mathrm{H}(5)$ & 0.9500 & $\mathrm{C}(29)-\mathrm{C}(30)$ & $1.393(3)$ \\
\hline $\mathrm{C}(6)-\mathrm{C}(8)$ & $1.502(3)$ & $\mathrm{C}(29)-\mathrm{H}(29)$ & 0.9500 \\
\hline $\mathrm{C}(7)-\mathrm{H}(7 \mathrm{~A})$ & 0.9900 & $\mathrm{C}(30)-\mathrm{C}(32)$ & $1.505(3)$ \\
\hline $\mathrm{C}(7)-\mathrm{H}(7 \mathrm{~B})$ & 0.9900 & $\mathrm{C}(31)-\mathrm{H}(31 \mathrm{~A})$ & 0.9900 \\
\hline $\mathrm{C}(8)-\mathrm{H}(8 \mathrm{~A})$ & 0.9900 & $\mathrm{C}(31)-\mathrm{H}(31 \mathrm{~B})$ & 0.9900 \\
\hline $\mathrm{C}(8)-\mathrm{H}(8 \mathrm{~B})$ & 0.9900 & $\mathrm{C}(32)-\mathrm{H}(32 \mathrm{~A})$ & 0.9900 \\
\hline $\mathrm{C}(9)-\mathrm{C}(12)$ & $1.529(3)$ & $\mathrm{C}(32)-\mathrm{H}(32 \mathrm{~B})$ & 0.9900 \\
\hline$C(9)-C(10)$ & $1.534(3)$ & $\mathrm{C}(33)-\mathrm{C}(36)$ & $1.533(3)$ \\
\hline $\mathrm{C}(9)-\mathrm{C}(11)$ & $1.537(3)$ & $C(33)-C(35)$ & $1.534(3)$ \\
\hline $\mathrm{C}(10)-\mathrm{H}(10 \mathrm{~A})$ & 0.9800 & $\mathrm{C}(33)-\mathrm{C}(34)$ & $1.542(3)$ \\
\hline $\mathrm{C}(10)-\mathrm{H}(10 \mathrm{~B})$ & 0.9800 & $\mathrm{C}(34)-\mathrm{H}(34 \mathrm{~A})$ & 0.9800 \\
\hline $\mathrm{C}(10)-\mathrm{H}(10 \mathrm{C})$ & 0.9800 & $\mathrm{C}(34)-\mathrm{H}(34 \mathrm{~B})$ & 0.9800 \\
\hline $\mathrm{C}(11)-\mathrm{H}(11 \mathrm{~A})$ & 0.9800 & $\mathrm{C}(34)-\mathrm{H}(34 \mathrm{C})$ & 0.9800 \\
\hline $\mathrm{C}(11)-\mathrm{H}(11 \mathrm{~B})$ & 0.9800 & $\mathrm{C}(35)-\mathrm{H}(35 \mathrm{~A})$ & 0.9800 \\
\hline $\mathrm{C}(11)-\mathrm{H}(11 \mathrm{C})$ & 0.9800 & $\mathrm{C}(35)-\mathrm{H}(35 \mathrm{~B})$ & 0.9800 \\
\hline $\mathrm{C}(12)-\mathrm{H}(12 \mathrm{~A})$ & 0.9800 & $\mathrm{C}(35)-\mathrm{H}(35 \mathrm{C})$ & 0.9800 \\
\hline $\mathrm{C}(12)-\mathrm{H}(12 \mathrm{~B})$ & 0.9800 & $\mathrm{C}(36)-\mathrm{H}(36 \mathrm{~A})$ & 0.9800 \\
\hline $\mathrm{C}(12)-\mathrm{H}(12 \mathrm{C})$ & 0.9800 & $\mathrm{C}(36)-\mathrm{H}(36 \mathrm{~B})$ & 0.9800 \\
\hline$C(13)-C(16)$ & $1.533(3)$ & $\mathrm{C}(36)-\mathrm{H}(36 \mathrm{C})$ & 0.9800 \\
\hline$C(13)-C(15)$ & $1.538(3)$ & $\mathrm{C}(37)-\mathrm{C}(38)$ & $1.531(3)$ \\
\hline $\mathrm{C}(13)-\mathrm{C}(14)$ & $1.539(3)$ & $C(37)-C(40)$ & $1.537(3)$ \\
\hline $\mathrm{C}(14)-\mathrm{H}(14 \mathrm{~A})$ & 0.9800 & $\mathrm{C}(37)-\mathrm{C}(39)$ & $1.540(3)$ \\
\hline $\mathrm{C}(14)-\mathrm{H}(14 \mathrm{~B})$ & 0.9800 & $\mathrm{C}(38)-\mathrm{H}(38 \mathrm{~A})$ & 0.9800 \\
\hline $\mathrm{C}(14)-\mathrm{H}(14 \mathrm{C})$ & 0.9800 & $\mathrm{C}(38)-\mathrm{H}(38 \mathrm{~B})$ & 0.9800 \\
\hline $\mathrm{C}(15)-\mathrm{H}(15 \mathrm{~A})$ & 0.9800 & $\mathrm{C}(38)-\mathrm{H}(38 \mathrm{C})$ & 0.9800 \\
\hline $\mathrm{C}(15)-\mathrm{H}(15 \mathrm{~B})$ & 0.9800 & $\mathrm{C}(39)-\mathrm{H}(39 \mathrm{~A})$ & 0.9800 \\
\hline $\mathrm{C}(15)-\mathrm{H}(15 \mathrm{C})$ & 0.9800 & $\mathrm{C}(39)-\mathrm{H}(39 \mathrm{~B})$ & 0.9800 \\
\hline $\mathrm{C}(16)-\mathrm{H}(16 \mathrm{~A})$ & 0.9800 & $\mathrm{C}(39)-\mathrm{H}(39 \mathrm{C})$ & 0.9800 \\
\hline $\mathrm{C}(16)-\mathrm{H}(16 \mathrm{~B})$ & 0.9800 & $\mathrm{C}(40)-\mathrm{H}(40 \mathrm{~A})$ & 0.9800 \\
\hline $\mathrm{C}(16)-\mathrm{H}(16 \mathrm{C})$ & 0.9800 & $\mathrm{C}(40)-\mathrm{H}(40 \mathrm{~B})$ & 0.9800 \\
\hline $\mathrm{C}(17)-\mathrm{C}(20)$ & $1.532(3)$ & $\mathrm{C}(40)-\mathrm{H}(40 \mathrm{C})$ & 0.9800 \\
\hline $\mathrm{C}(17)-\mathrm{C}(18)$ & $1.538(3)$ & $\mathrm{C}(41)-\mathrm{C}(43)$ & $1.529(3)$ \\
\hline
\end{tabular}




\begin{tabular}{|c|c|}
\hline$C(41)-C(44)$ & $1.531(3)$ \\
\hline C(41)-C(42) & $1.538(3)$ \\
\hline $\mathrm{C}(42)-\mathrm{H}(42 \mathrm{~A})$ & 0.9800 \\
\hline $\mathrm{C}(42)-\mathrm{H}(42 \mathrm{~B})$ & 0.9800 \\
\hline $\mathrm{C}(42)-\mathrm{H}(42 \mathrm{C})$ & 0.9800 \\
\hline $\mathrm{C}(43)-\mathrm{H}(43 \mathrm{~A})$ & 0.9800 \\
\hline $\mathrm{C}(43)-\mathrm{H}(43 \mathrm{~B})$ & 0.9800 \\
\hline $\mathrm{C}(43)-\mathrm{H}(43 \mathrm{C})$ & 0.9800 \\
\hline $\mathrm{C}(44)-\mathrm{H}(44 \mathrm{~A})$ & 0.9800 \\
\hline $\mathrm{C}(44)-\mathrm{H}(44 \mathrm{~B})$ & 0.9800 \\
\hline $\mathrm{C}(44)-\mathrm{H}(44 \mathrm{C})$ & 0.9800 \\
\hline$C(45)-C(47)$ & $1.533(3)$ \\
\hline$C(45)-C(48)$ & $1.538(3)$ \\
\hline $\mathrm{C}(45)-\mathrm{C}(46)$ & $1.543(3)$ \\
\hline $\mathrm{C}(46)-\mathrm{H}(46 \mathrm{~A})$ & 0.9800 \\
\hline $\mathrm{C}(46)-\mathrm{H}(46 \mathrm{~B})$ & 0.9800 \\
\hline $\mathrm{C}(46)-\mathrm{H}(46 \mathrm{C})$ & 0.9800 \\
\hline $\mathrm{N}(1)-\operatorname{Ir}(1)-\mathrm{C}(1)$ & $179.29(6)$ \\
\hline $\mathrm{N}(1)-\operatorname{Ir}(1)-\mathrm{P}(1)$ & $100.35(4)$ \\
\hline $\mathrm{C}(1)-\operatorname{Ir}(1)-\mathrm{P}(1)$ & $80.15(5)$ \\
\hline $\mathrm{N}(1)-\operatorname{Ir}(1)-\mathrm{P}(2)$ & $99.00(4)$ \\
\hline $\mathrm{C}(1)-\operatorname{Ir}(1)-\mathrm{P}(2)$ & $80.49(5)$ \\
\hline $\mathrm{P}(1)-\operatorname{Ir}(1)-\mathrm{P}(2)$ & $160.630(16)$ \\
\hline $\mathrm{N}(2)-\operatorname{Ir}(2)-\mathrm{C}(25)$ & $179.57(7)$ \\
\hline $\mathrm{N}(2)-\operatorname{Ir}(2)-\mathrm{P}(3)$ & $99.07(4)$ \\
\hline $\mathrm{C}(25)-\operatorname{Ir}(2)-\mathrm{P}(3)$ & $80.50(5)$ \\
\hline$N(2)-\operatorname{Ir}(2)-P(4)$ & $100.28(4)$ \\
\hline $\mathrm{C}(25)-\operatorname{Ir}(2)-\mathrm{P}(4)$ & $80.14(5)$ \\
\hline $\mathrm{P}(3)-\operatorname{Ir}(2)-\mathrm{P}(4)$ & $160.623(16)$ \\
\hline $\mathrm{N}(2)-\mathrm{N}(1)-\operatorname{Ir}(1)$ & $178.97(15)$ \\
\hline $\mathrm{N}(1)-\mathrm{N}(2)-\operatorname{Ir}(2)$ & $179.64(16)$ \\
\hline$C(7)-P(1)-C(13)$ & $102.51(9)$ \\
\hline $\mathrm{C}(7)-\mathrm{P}(1)-\mathrm{C}(9)$ & $103.61(9)$ \\
\hline $\mathrm{C}(13)-\mathrm{P}(1)-\mathrm{C}(9)$ & $110.66(8)$ \\
\hline $\mathrm{C}(7)-\mathrm{P}(1)-\operatorname{Ir}(1)$ & $103.03(6)$ \\
\hline $\mathrm{C}(13)-\mathrm{P}(1)-\operatorname{Ir}(1)$ & $119.71(6)$ \\
\hline $\mathrm{C}(9)-\mathrm{P}(1)-\operatorname{Ir}(1)$ & $114.75(6)$ \\
\hline $\mathrm{C}(8)-\mathrm{P}(2)-\mathrm{C}(17)$ & $101.86(8)$ \\
\hline $\mathrm{C}(8)-\mathrm{P}(2)-\mathrm{C}(21)$ & $103.48(9)$ \\
\hline $\mathrm{C}(17)-\mathrm{P}(2)-\mathrm{C}(21)$ & $111.36(8)$ \\
\hline $\mathrm{C}(8)-\mathrm{P}(2)-\operatorname{Ir}(1)$ & $102.54(6)$ \\
\hline$C(17)-P(2)-\operatorname{Ir}(1)$ & $118.87(6)$ \\
\hline $\mathrm{C}(21)-\mathrm{P}(2)-\operatorname{Ir}(1)$ & $115.84(6)$ \\
\hline $\mathrm{C}(31)-\mathrm{P}(3)-\mathrm{C}(33)$ & $101.92(8)$ \\
\hline $\mathrm{C}(31)-\mathrm{P}(3)-\mathrm{C}(37)$ & $103.51(8)$ \\
\hline $\mathrm{C}(33)-\mathrm{P}(3)-\mathrm{C}(37)$ & $110.75(9)$ \\
\hline $\mathrm{C}(31)-\mathrm{P}(3)-\operatorname{Ir}(2)$ & $102.68(6)$ \\
\hline $\mathrm{C}(33)-\mathrm{P}(3)-\operatorname{Ir}(2)$ & $120.41(6)$ \\
\hline $\mathrm{C}(37)-\mathrm{P}(3)-\operatorname{Ir}(2)$ & $114.74(6)$ \\
\hline $\mathrm{C}(32)-\mathrm{P}(4)-\mathrm{C}(45)$ & $102.25(8)$ \\
\hline $\mathrm{C}(32)-\mathrm{P}(4)-\mathrm{C}(41)$ & $103.09(9)$ \\
\hline $\mathrm{C}(45)-\mathrm{P}(4)-\mathrm{C}(41)$ & $111.14(8)$ \\
\hline$C(32)-P(4)-\operatorname{Ir}(2)$ & $102.88(6)$ \\
\hline $\mathrm{C}(45)-\mathrm{P}(4)-\operatorname{Ir}(2)$ & $118.64(6)$ \\
\hline $\mathrm{C}(41)-\mathrm{P}(4)-\operatorname{Ir}(2)$ & $116.03(6)$ \\
\hline$C(2)-C(1)-C(6)$ & $115.38(16)$ \\
\hline$C(2)-C(1)-\operatorname{Ir}(1)$ & $122.52(14)$ \\
\hline$C(6)-C(1)-\operatorname{Ir}(1)$ & $122.10(13)$ \\
\hline $\mathrm{C}(3)-\mathrm{C}(2)-\mathrm{C}(1)$ & $122.28(17)$ \\
\hline $\mathrm{C}(3)-\mathrm{C}(2)-\mathrm{C}(7)$ & $119.70(17)$ \\
\hline $\mathrm{C}(1)-\mathrm{C}(2)-\mathrm{C}(7)$ & $118.01(16)$ \\
\hline$C(4)-C(3)-C(2)$ & $120.47(18)$ \\
\hline $\mathrm{C}(4)-\mathrm{C}(3)-\mathrm{H}(3)$ & 119.8 \\
\hline
\end{tabular}

$\begin{array}{ll}\mathrm{C}(47)-\mathrm{H}(47 \mathrm{~A}) & 0.9800 \\ \mathrm{C}(47)-\mathrm{H}(47 \mathrm{~B}) & 0.9800 \\ \mathrm{C}(47)-\mathrm{H}(47 \mathrm{C}) & 0.9800 \\ \mathrm{C}(48)-\mathrm{H}(48 \mathrm{~A}) & 0.9800 \\ \mathrm{C}(48)-\mathrm{H}(48 \mathrm{~B}) & 0.9800 \\ \mathrm{C}(48)-\mathrm{H}(48 \mathrm{C}) & 0.9800 \\ \mathrm{C}(49)-\mathrm{C}(50) & 1.500(4) \\ \mathrm{C}(49)-\mathrm{C}(49) \# 1 & 1.556(7) \\ \mathrm{C}(49)-\mathrm{H}(49 \mathrm{~A}) & 0.9900 \\ \mathrm{C}(49)-\mathrm{H}(49 \mathrm{~B}) & 0.9900 \\ \mathrm{C}(50)-\mathrm{C}(51) & 1.513(5) \\ \mathrm{C}(50)-\mathrm{H}(50 \mathrm{~A}) & 0.9900 \\ \mathrm{C}(50)-\mathrm{H}(50 \mathrm{~B}) & 0.9900 \\ \mathrm{C}(51)-\mathrm{H}(51 \mathrm{~A}) & 0.9800 \\ \mathrm{C}(51)-\mathrm{H}(51 \mathrm{~B}) & 0.9800 \\ \mathrm{C}(51)-\mathrm{H}(51 \mathrm{C}) & 0.9800\end{array}$

$\mathrm{C}(2)-\mathrm{C}(3)-\mathrm{H}(3) \quad 119.8$

$\mathrm{C}(5)-\mathrm{C}(4)-\mathrm{C}(3) \quad 118.98(17)$

$\mathrm{C}(5)-\mathrm{C}(4)-\mathrm{H}(4) \quad 120.5$

$\mathrm{C}(3)-\mathrm{C}(4)-\mathrm{H}(4) \quad 120.5$

$\mathrm{C}(4)-\mathrm{C}(5)-\mathrm{C}(6) \quad 120.43(18)$

$\mathrm{C}(4)-\mathrm{C}(5)-\mathrm{H}(5) \quad 119.8$

$\mathrm{C}(6)-\mathrm{C}(5)-\mathrm{H}(5) \quad 119.8$

$\mathrm{C}(5)-\mathrm{C}(6)-\mathrm{C}(1) \quad 122.44(17)$

$\mathrm{C}(5)-\mathrm{C}(6)-\mathrm{C}(8) \quad 119.61(16)$

$\mathrm{C}(1)-\mathrm{C}(6)-\mathrm{C}(8)$

$\mathrm{C}(2)-\mathrm{C}(7)-\mathrm{P}(1) \quad 107.07(13)$

$\mathrm{C}(2)-\mathrm{C}(7)-\mathrm{H}(7 \mathrm{~A}) \quad 110.3$

$\mathrm{P}(1)-\mathrm{C}(7)-\mathrm{H}(7 \mathrm{~A}) \quad 110.3$

$\mathrm{C}(2)-\mathrm{C}(7)-\mathrm{H}(7 \mathrm{~B}) \quad 110.3$

$\mathrm{P}(1)-\mathrm{C}(7)-\mathrm{H}(7 \mathrm{~B}) \quad 110.3$

$\mathrm{H}(7 \mathrm{~A})-\mathrm{C}(7)-\mathrm{H}(7 \mathrm{~B})$

$\mathrm{C}(6)-\mathrm{C}(8)-\mathrm{P}(2) \quad 107.31(12)$

$\mathrm{C}(6)-\mathrm{C}(8)-\mathrm{H}(8 \mathrm{~A})$

$\mathrm{P}(2)-\mathrm{C}(8)-\mathrm{H}(8 \mathrm{~A}) \quad 110.3$

$\mathrm{C}(6)-\mathrm{C}(8)-\mathrm{H}(8 \mathrm{~B}) \quad 110.3$

$\mathrm{P}(2)-\mathrm{C}(8)-\mathrm{H}(8 \mathrm{~B}) \quad 110.3$

$\mathrm{H}(8 \mathrm{~A})-\mathrm{C}(8)-\mathrm{H}(8 \mathrm{~B}) \quad 108.5$

$\mathrm{C}(12)-\mathrm{C}(9)-\mathrm{C}(10) \quad 109.89(17)$

$\mathrm{C}(12)-\mathrm{C}(9)-\mathrm{C}(11) \quad 107.35(16)$

$\mathrm{C}(10)-\mathrm{C}(9)-\mathrm{C}(11) \quad 108.29(15)$

$\mathrm{C}(12)-\mathrm{C}(9)-\mathrm{P}(1) \quad 108.37(13)$

$\mathrm{C}(10)-\mathrm{C}(9)-\mathrm{P}(1) \quad 114.61(14)$

$\mathrm{C}(11)-\mathrm{C}(9)-\mathrm{P}(1) \quad 108.07(13)$

$\mathrm{C}(9)-\mathrm{C}(10)-\mathrm{H}(10 \mathrm{~A}) \quad 109.5$

$\mathrm{C}(9)-\mathrm{C}(10)-\mathrm{H}(10 \mathrm{~B}) \quad 109.5$

$\mathrm{H}(10 \mathrm{~A})-\mathrm{C}(10)-\mathrm{H}(10 \mathrm{~B}) \quad 109.5$

$\mathrm{C}(9)-\mathrm{C}(10)-\mathrm{H}(10 \mathrm{C}) \quad 109.5$

$\mathrm{H}(10 \mathrm{~A})-\mathrm{C}(10)-\mathrm{H}(10 \mathrm{C}) \quad 109.5$

$\mathrm{H}(10 \mathrm{~B})-\mathrm{C}(10)-\mathrm{H}(10 \mathrm{C}) \quad 109.5$

$\mathrm{C}(9)-\mathrm{C}(11)-\mathrm{H}(11 \mathrm{~A}) \quad 109.5$

$\mathrm{C}(9)-\mathrm{C}(11)-\mathrm{H}(11 \mathrm{~B}) \quad 109.5$

$\mathrm{H}(11 \mathrm{~A})-\mathrm{C}(11)-\mathrm{H}(11 \mathrm{~B}) \quad 109.5$

$\mathrm{C}(9)-\mathrm{C}(11)-\mathrm{H}(11 \mathrm{C}) \quad 109.5$

$\mathrm{H}(11 \mathrm{~A})-\mathrm{C}(11)-\mathrm{H}(11 \mathrm{C}) \quad 109.5$

$\mathrm{H}(11 \mathrm{~B})-\mathrm{C}(11)-\mathrm{H}(1 \mathrm{C}) \quad 109.5$

$\mathrm{C}(9)-\mathrm{C}(12)-\mathrm{H}(12 \mathrm{~A}) \quad 109.5$

$\mathrm{C}(9)-\mathrm{C}(12)-\mathrm{H}(12 \mathrm{~B}) \quad 109.5$

$\mathrm{H}(12 \mathrm{~A})-\mathrm{C}(12)-\mathrm{H}(12 \mathrm{~B}) \quad 109.5$

$\mathrm{C}(9)-\mathrm{C}(12)-\mathrm{H}(12 \mathrm{C}) \quad 109.5$

$\mathrm{H}(12 \mathrm{~A})-\mathrm{C}(12)-\mathrm{H}(12 \mathrm{C}) \quad 109.5$

$\mathrm{H}(12 \mathrm{~B})-\mathrm{C}(12)-\mathrm{H}(12 \mathrm{C}) \quad 109.5$ 


\begin{tabular}{|c|c|}
\hline$C(16)-C(13)-C(15)$ & $108.47(16)$ \\
\hline$C(16)-C(13)-C(14)$ & $109.73(17)$ \\
\hline$C(15)-C(13)-C(14)$ & $107.55(17)$ \\
\hline $\mathrm{C}(16)-\mathrm{C}(13)-\mathrm{P}(1)$ & $110.81(13)$ \\
\hline $\mathrm{C}(15)-\mathrm{C}(13)-\mathrm{P}(1)$ & $105.12(13)$ \\
\hline $\mathrm{C}(14)-\mathrm{C}(13)-\mathrm{P}(1)$ & $114.84(14)$ \\
\hline $\mathrm{C}(13)-\mathrm{C}(14)-\mathrm{H}(14 \mathrm{~A})$ & 109.5 \\
\hline $\mathrm{C}(13)-\mathrm{C}(14)-\mathrm{H}(14 \mathrm{~B})$ & 109.5 \\
\hline $\mathrm{H}(14 \mathrm{~A})-\mathrm{C}(14)-\mathrm{H}(14 \mathrm{~B})$ & 109.5 \\
\hline $\mathrm{C}(13)-\mathrm{C}(14)-\mathrm{H}(14 \mathrm{C})$ & 109.5 \\
\hline $\mathrm{H}(14 \mathrm{~A})-\mathrm{C}(14)-\mathrm{H}(14 \mathrm{C})$ & 109.5 \\
\hline $\mathrm{H}(14 \mathrm{~B})-\mathrm{C}(14)-\mathrm{H}(14 \mathrm{C})$ & 109.5 \\
\hline $\mathrm{C}(13)-\mathrm{C}(15)-\mathrm{H}(15 \mathrm{~A})$ & 109.5 \\
\hline $\mathrm{C}(13)-\mathrm{C}(15)-\mathrm{H}(15 \mathrm{~B})$ & 109.5 \\
\hline $\mathrm{H}(15 \mathrm{~A})-\mathrm{C}(15)-\mathrm{H}(15 \mathrm{~B})$ & 109.5 \\
\hline $\mathrm{C}(13)-\mathrm{C}(15)-\mathrm{H}(15 \mathrm{C})$ & 109.5 \\
\hline $\mathrm{H}(15 \mathrm{~A})-\mathrm{C}(15)-\mathrm{H}(15 \mathrm{C})$ & 109.5 \\
\hline $\mathrm{H}(15 \mathrm{~B})-\mathrm{C}(15)-\mathrm{H}(15 \mathrm{C})$ & 109.5 \\
\hline $\mathrm{C}(13)-\mathrm{C}(16)-\mathrm{H}(16 \mathrm{~A})$ & 109.5 \\
\hline $\mathrm{C}(13)-\mathrm{C}(16)-\mathrm{H}(16 \mathrm{~B})$ & 109.5 \\
\hline $\mathrm{H}(16 \mathrm{~A})-\mathrm{C}(16)-\mathrm{H}(16 \mathrm{~B})$ & 109.5 \\
\hline $\mathrm{C}(13)-\mathrm{C}(16)-\mathrm{H}(16 \mathrm{C})$ & 109.5 \\
\hline $\mathrm{H}(16 \mathrm{~A})-\mathrm{C}(16)-\mathrm{H}(16 \mathrm{C})$ & 109.5 \\
\hline $\mathrm{H}(16 \mathrm{~B})-\mathrm{C}(16)-\mathrm{H}(16 \mathrm{C})$ & 109.5 \\
\hline$C(20)-C(17)-C(18)$ & $108.52(15)$ \\
\hline $\mathrm{C}(20)-\mathrm{C}(17)-\mathrm{C}(19)$ & $109.61(15)$ \\
\hline $\mathrm{C}(18)-\mathrm{C}(17)-\mathrm{C}(19)$ & $107.82(15)$ \\
\hline $\mathrm{C}(20)-\mathrm{C}(17)-\mathrm{P}(2)$ & $110.93(13)$ \\
\hline $\mathrm{C}(18)-\mathrm{C}(17)-\mathrm{P}(2)$ & $105.36(12)$ \\
\hline $\mathrm{C}(19)-\mathrm{C}(17)-\mathrm{P}(2)$ & $114.33(13)$ \\
\hline $\mathrm{C}(17)-\mathrm{C}(18)-\mathrm{H}(18 \mathrm{~A})$ & 109.5 \\
\hline $\mathrm{C}(17)-\mathrm{C}(18)-\mathrm{H}(18 \mathrm{~B})$ & 109.5 \\
\hline $\mathrm{H}(18 \mathrm{~A})-\mathrm{C}(18)-\mathrm{H}(18 \mathrm{~B})$ & 109.5 \\
\hline $\mathrm{C}(17)-\mathrm{C}(18)-\mathrm{H}(18 \mathrm{C})$ & 109.5 \\
\hline $\mathrm{H}(18 \mathrm{~A})-\mathrm{C}(18)-\mathrm{H}(18 \mathrm{C})$ & 109.5 \\
\hline $\mathrm{H}(18 \mathrm{~B})-\mathrm{C}(18)-\mathrm{H}(18 \mathrm{C})$ & 109.5 \\
\hline $\mathrm{C}(17)-\mathrm{C}(19)-\mathrm{H}(19 \mathrm{~A})$ & 109.5 \\
\hline $\mathrm{C}(17)-\mathrm{C}(19)-\mathrm{H}(19 \mathrm{~B})$ & 109.5 \\
\hline $\mathrm{H}(19 \mathrm{~A})-\mathrm{C}(19)-\mathrm{H}(19 \mathrm{~B})$ & 109.5 \\
\hline $\mathrm{C}(17)-\mathrm{C}(19)-\mathrm{H}(19 \mathrm{C})$ & 109.5 \\
\hline $\mathrm{H}(19 \mathrm{~A})-\mathrm{C}(19)-\mathrm{H}(19 \mathrm{C})$ & 109.5 \\
\hline $\mathrm{H}(19 \mathrm{~B})-\mathrm{C}(19)-\mathrm{H}(19 \mathrm{C})$ & 109.5 \\
\hline $\mathrm{C}(17)-\mathrm{C}(20)-\mathrm{H}(20 \mathrm{~A})$ & 109.5 \\
\hline $\mathrm{C}(17)-\mathrm{C}(20)-\mathrm{H}(20 \mathrm{~B})$ & 109.5 \\
\hline $\mathrm{H}(20 \mathrm{~A})-\mathrm{C}(20)-\mathrm{H}(20 \mathrm{~B})$ & 109.5 \\
\hline $\mathrm{C}(17)-\mathrm{C}(20)-\mathrm{H}(20 \mathrm{C})$ & 109.5 \\
\hline $\mathrm{H}(20 \mathrm{~A})-\mathrm{C}(20)-\mathrm{H}(20 \mathrm{C})$ & 109.5 \\
\hline $\mathrm{H}(20 \mathrm{~B})-\mathrm{C}(20)-\mathrm{H}(20 \mathrm{C})$ & 109.5 \\
\hline$C(24)-C(21)-C(23)$ & $110.02(16)$ \\
\hline $\mathrm{C}(24)-\mathrm{C}(21)-\mathrm{C}(22)$ & $107.41(17)$ \\
\hline$C(23)-C(21)-C(22)$ & $108.82(16)$ \\
\hline $\mathrm{C}(24)-\mathrm{C}(21)-\mathrm{P}(2)$ & $108.65(13)$ \\
\hline $\mathrm{C}(23)-\mathrm{C}(21)-\mathrm{P}(2)$ & $114.52(14)$ \\
\hline $\mathrm{C}(22)-\mathrm{C}(21)-\mathrm{P}(2)$ & $107.16(13)$ \\
\hline $\mathrm{C}(21)-\mathrm{C}(22)-\mathrm{H}(22 \mathrm{~A})$ & 109.5 \\
\hline $\mathrm{C}(21)-\mathrm{C}(22)-\mathrm{H}(22 \mathrm{~B})$ & 109.5 \\
\hline $\mathrm{H}(22 \mathrm{~A})-\mathrm{C}(22)-\mathrm{H}(22 \mathrm{~B})$ & 109.5 \\
\hline $\mathrm{C}(21)-\mathrm{C}(22)-\mathrm{H}(22 \mathrm{C})$ & 109.5 \\
\hline $\mathrm{H}(22 \mathrm{~A})-\mathrm{C}(22)-\mathrm{H}(22 \mathrm{C})$ & 109.5 \\
\hline $\mathrm{H}(22 \mathrm{~B})-\mathrm{C}(22)-\mathrm{H}(22 \mathrm{C})$ & 109.5 \\
\hline $\mathrm{C}(21)-\mathrm{C}(23)-\mathrm{H}(23 \mathrm{~A})$ & 109.5 \\
\hline $\mathrm{C}(21)-\mathrm{C}(23)-\mathrm{H}(23 \mathrm{~B})$ & 109.5 \\
\hline $\mathrm{H}(23 \mathrm{~A})-\mathrm{C}(23)-\mathrm{H}(23 \mathrm{~B})$ & 109.5 \\
\hline $\mathrm{C}(21)-\mathrm{C}(23)-\mathrm{H}(23 \mathrm{C})$ & 109.5 \\
\hline
\end{tabular}

\begin{tabular}{|c|c|}
\hline $\mathrm{H}(23 \mathrm{~A})-\mathrm{C}(23)-\mathrm{H}(23 \mathrm{C})$ & 109.5 \\
\hline $\mathrm{H}(23 \mathrm{~B})-\mathrm{C}(23)-\mathrm{H}(23 \mathrm{C})$ & 109.5 \\
\hline $\mathrm{C}(21)-\mathrm{C}(24)-\mathrm{H}(24 \mathrm{~A})$ & 109.5 \\
\hline $\mathrm{C}(21)-\mathrm{C}(24)-\mathrm{H}(24 \mathrm{~B})$ & 109.5 \\
\hline $\mathrm{H}(24 \mathrm{~A})-\mathrm{C}(24)-\mathrm{H}(24 \mathrm{~B})$ & 109.5 \\
\hline $\mathrm{C}(21)-\mathrm{C}(24)-\mathrm{H}(24 \mathrm{C})$ & 109.5 \\
\hline $\mathrm{H}(24 \mathrm{~A})-\mathrm{C}(24)-\mathrm{H}(24 \mathrm{C})$ & 109.5 \\
\hline $\mathrm{H}(24 \mathrm{~B})-\mathrm{C}(24)-\mathrm{H}(24 \mathrm{C})$ & 109.5 \\
\hline$C(30)-C(25)-C(26)$ & $115.19(16)$ \\
\hline$C(30)-C(25)-\operatorname{Ir}(2)$ & $122.68(13)$ \\
\hline $\mathrm{C}(26)-\mathrm{C}(25)-\operatorname{Ir}(2)$ & $122.13(13)$ \\
\hline $\mathrm{C}(27)-\mathrm{C}(26)-\mathrm{C}(25)$ & $122.35(17)$ \\
\hline $\mathrm{C}(27)-\mathrm{C}(26)-\mathrm{C}(31)$ & $119.76(16)$ \\
\hline $\mathrm{C}(25)-\mathrm{C}(26)-\mathrm{C}(31)$ & $117.88(16)$ \\
\hline $\mathrm{C}(28)-\mathrm{C}(27)-\mathrm{C}(26)$ & $120.45(17)$ \\
\hline $\mathrm{C}(28)-\mathrm{C}(27)-\mathrm{H}(27)$ & 119.8 \\
\hline $\mathrm{C}(26)-\mathrm{C}(27)-\mathrm{H}(27)$ & 119.8 \\
\hline $\mathrm{C}(29)-\mathrm{C}(28)-\mathrm{C}(27)$ & $118.69(17)$ \\
\hline $\mathrm{C}(29)-\mathrm{C}(28)-\mathrm{H}(28)$ & 120.7 \\
\hline $\mathrm{C}(27)-\mathrm{C}(28)-\mathrm{H}(28)$ & 120.7 \\
\hline $\mathrm{C}(28)-\mathrm{C}(29)-\mathrm{C}(30)$ & $120.83(18)$ \\
\hline $\mathrm{C}(28)-\mathrm{C}(29)-\mathrm{H}(29)$ & 119.6 \\
\hline $\mathrm{C}(30)-\mathrm{C}(29)-\mathrm{H}(29)$ & 119.6 \\
\hline$C(29)-C(30)-C(25)$ & $122.44(17)$ \\
\hline $\mathrm{C}(29)-\mathrm{C}(30)-\mathrm{C}(32)$ & $119.97(17)$ \\
\hline $\mathrm{C}(25)-\mathrm{C}(30)-\mathrm{C}(32)$ & $117.59(16)$ \\
\hline $\mathrm{C}(26)-\mathrm{C}(31)-\mathrm{P}(3)$ & $107.14(12)$ \\
\hline $\mathrm{C}(26)-\mathrm{C}(31)-\mathrm{H}(31 \mathrm{~A})$ & 110.3 \\
\hline $\mathrm{P}(3)-\mathrm{C}(31)-\mathrm{H}(31 \mathrm{~A})$ & 110.3 \\
\hline $\mathrm{C}(26)-\mathrm{C}(31)-\mathrm{H}(31 \mathrm{~B})$ & 110.3 \\
\hline $\mathrm{P}(3)-\mathrm{C}(31)-\mathrm{H}(31 \mathrm{~B})$ & 110.3 \\
\hline $\mathrm{H}(31 \mathrm{~A})-\mathrm{C}(31)-\mathrm{H}(31 \mathrm{~B})$ & 108.5 \\
\hline $\mathrm{C}(30)-\mathrm{C}(32)-\mathrm{P}(4)$ & $107.24(12)$ \\
\hline $\mathrm{C}(30)-\mathrm{C}(32)-\mathrm{H}(32 \mathrm{~A})$ & 110.3 \\
\hline $\mathrm{P}(4)-\mathrm{C}(32)-\mathrm{H}(32 \mathrm{~A})$ & 110.3 \\
\hline $\mathrm{C}(30)-\mathrm{C}(32)-\mathrm{H}(32 \mathrm{~B})$ & 110.3 \\
\hline $\mathrm{P}(4)-\mathrm{C}(32)-\mathrm{H}(32 \mathrm{~B})$ & 110.3 \\
\hline $\mathrm{H}(32 \mathrm{~A})-\mathrm{C}(32)-\mathrm{H}(32 \mathrm{~B})$ & 108.5 \\
\hline$C(36)-C(33)-C(35)$ & $108.64(16)$ \\
\hline$C(36)-C(33)-C(34)$ & $109.18(17)$ \\
\hline $\mathrm{C}(35)-\mathrm{C}(33)-\mathrm{C}(34)$ & $107.83(17)$ \\
\hline $\mathrm{C}(36)-\mathrm{C}(33)-\mathrm{P}(3)$ & $111.79(14)$ \\
\hline $\mathrm{C}(35)-\mathrm{C}(33)-\mathrm{P}(3)$ & $104.71(13)$ \\
\hline $\mathrm{C}(34)-\mathrm{C}(33)-\mathrm{P}(3)$ & $114.41(14)$ \\
\hline $\mathrm{C}(33)-\mathrm{C}(34)-\mathrm{H}(34 \mathrm{~A})$ & 109.5 \\
\hline $\mathrm{C}(33)-\mathrm{C}(34)-\mathrm{H}(34 \mathrm{~B})$ & 109.5 \\
\hline $\mathrm{H}(34 \mathrm{~A})-\mathrm{C}(34)-\mathrm{H}(34 \mathrm{~B})$ & 109.5 \\
\hline $\mathrm{C}(33)-\mathrm{C}(34)-\mathrm{H}(34 \mathrm{C})$ & 109.5 \\
\hline $\mathrm{H}(34 \mathrm{~A})-\mathrm{C}(34)-\mathrm{H}(34 \mathrm{C})$ & 109.5 \\
\hline $\mathrm{H}(34 \mathrm{~B})-\mathrm{C}(34)-\mathrm{H}(34 \mathrm{C})$ & 109.5 \\
\hline $\mathrm{C}(33)-\mathrm{C}(35)-\mathrm{H}(35 \mathrm{~A})$ & 109.5 \\
\hline $\mathrm{C}(33)-\mathrm{C}(35)-\mathrm{H}(35 \mathrm{~B})$ & 109.5 \\
\hline $\mathrm{H}(35 \mathrm{~A})-\mathrm{C}(35)-\mathrm{H}(35 \mathrm{~B})$ & 109.5 \\
\hline $\mathrm{C}(33)-\mathrm{C}(35)-\mathrm{H}(35 \mathrm{C})$ & 109.5 \\
\hline $\mathrm{H}(35 \mathrm{~A})-\mathrm{C}(35)-\mathrm{H}(35 \mathrm{C})$ & 109.5 \\
\hline $\mathrm{H}(35 \mathrm{~B})-\mathrm{C}(35)-\mathrm{H}(35 \mathrm{C})$ & 109.5 \\
\hline $\mathrm{C}(33)-\mathrm{C}(36)-\mathrm{H}(36 \mathrm{~A})$ & 109.5 \\
\hline $\mathrm{C}(33)-\mathrm{C}(36)-\mathrm{H}(36 \mathrm{~B})$ & 109.5 \\
\hline $\mathrm{H}(36 \mathrm{~A})-\mathrm{C}(36)-\mathrm{H}(36 \mathrm{~B})$ & 109.5 \\
\hline $\mathrm{C}(33)-\mathrm{C}(36)-\mathrm{H}(36 \mathrm{C})$ & 109.5 \\
\hline $\mathrm{H}(36 \mathrm{~A})-\mathrm{C}(36)-\mathrm{H}(36 \mathrm{C})$ & 109.5 \\
\hline $\mathrm{H}(36 \mathrm{~B})-\mathrm{C}(36)-\mathrm{H}(36 \mathrm{C})$ & 109.5 \\
\hline$C(38)-C(37)-C(40)$ & $110.07(17)$ \\
\hline $\mathrm{C}(38)-\mathrm{C}(37)-\mathrm{C}(39)$ & $108.02(16)$ \\
\hline
\end{tabular}




\begin{tabular}{|c|c|c|c|}
\hline $\mathrm{C}(40)-\mathrm{C}(37)-\mathrm{C}(39)$ & $107.68(18)$ & $\mathrm{H}(44 \mathrm{~A})-\mathrm{C}(44)-\mathrm{H}(44 \mathrm{C})$ & 109.5 \\
\hline $\mathrm{C}(38)-\mathrm{C}(37)-\mathrm{P}(3)$ & $114.25(14)$ & $\mathrm{H}(44 \mathrm{~B})-\mathrm{C}(44)-\mathrm{H}(44 \mathrm{C})$ & 109.5 \\
\hline $\mathrm{C}(40)-\mathrm{C}(37)-\mathrm{P}(3)$ & $108.34(13)$ & $\mathrm{C}(47)-\mathrm{C}(45)-\mathrm{C}(48)$ & $109.03(16)$ \\
\hline $\mathrm{C}(39)-\mathrm{C}(37)-\mathrm{P}(3)$ & $108.27(13)$ & $C(47)-C(45)-C(46)$ & $109.18(15)$ \\
\hline $\mathrm{C}(37)-\mathrm{C}(38)-\mathrm{H}(38 \mathrm{~A})$ & 109.5 & $\mathrm{C}(48)-\mathrm{C}(45)-\mathrm{C}(46)$ & $107.71(16)$ \\
\hline $\mathrm{C}(37)-\mathrm{C}(38)-\mathrm{H}(38 \mathrm{~B})$ & 109.5 & $\mathrm{C}(47)-\mathrm{C}(45)-\mathrm{P}(4)$ & $110.35(13)$ \\
\hline $\mathrm{H}(38 \mathrm{~A})-\mathrm{C}(38)-\mathrm{H}(38 \mathrm{~B})$ & 109.5 & $\mathrm{C}(48)-\mathrm{C}(45)-\mathrm{P}(4)$ & $105.72(13)$ \\
\hline $\mathrm{C}(37)-\mathrm{C}(38)-\mathrm{H}(38 \mathrm{C})$ & 109.5 & $\mathrm{C}(46)-\mathrm{C}(45)-\mathrm{P}(4)$ & $114.66(13)$ \\
\hline $\mathrm{H}(38 \mathrm{~A})-\mathrm{C}(38)-\mathrm{H}(38 \mathrm{C})$ & 109.5 & $\mathrm{C}(45)-\mathrm{C}(46)-\mathrm{H}(46 \mathrm{~A})$ & 109.5 \\
\hline $\mathrm{H}(38 \mathrm{~B})-\mathrm{C}(38)-\mathrm{H}(38 \mathrm{C})$ & 109.5 & $\mathrm{C}(45)-\mathrm{C}(46)-\mathrm{H}(46 \mathrm{~B})$ & 109.5 \\
\hline $\mathrm{C}(37)-\mathrm{C}(39)-\mathrm{H}(39 \mathrm{~A})$ & 109.5 & $\mathrm{H}(46 \mathrm{~A})-\mathrm{C}(46)-\mathrm{H}(46 \mathrm{~B})$ & 109.5 \\
\hline $\mathrm{C}(37)-\mathrm{C}(39)-\mathrm{H}(39 \mathrm{~B})$ & 109.5 & $\mathrm{C}(45)-\mathrm{C}(46)-\mathrm{H}(46 \mathrm{C})$ & 109.5 \\
\hline $\mathrm{H}(39 \mathrm{~A})-\mathrm{C}(39)-\mathrm{H}(39 \mathrm{~B})$ & 109.5 & $\mathrm{H}(46 \mathrm{~A})-\mathrm{C}(46)-\mathrm{H}(46 \mathrm{C})$ & 109.5 \\
\hline $\mathrm{C}(37)-\mathrm{C}(39)-\mathrm{H}(39 \mathrm{C})$ & 109.5 & $\mathrm{H}(46 \mathrm{~B})-\mathrm{C}(46)-\mathrm{H}(46 \mathrm{C})$ & 109.5 \\
\hline $\mathrm{H}(39 \mathrm{~A})-\mathrm{C}(39)-\mathrm{H}(39 \mathrm{C})$ & 109.5 & $\mathrm{C}(45)-\mathrm{C}(47)-\mathrm{H}(47 \mathrm{~A})$ & 109.5 \\
\hline $\mathrm{H}(39 \mathrm{~B})-\mathrm{C}(39)-\mathrm{H}(39 \mathrm{C})$ & 109.5 & $\mathrm{C}(45)-\mathrm{C}(47)-\mathrm{H}(47 \mathrm{~B})$ & 109.5 \\
\hline $\mathrm{C}(37)-\mathrm{C}(40)-\mathrm{H}(40 \mathrm{~A})$ & 109.5 & $\mathrm{H}(47 \mathrm{~A})-\mathrm{C}(47)-\mathrm{H}(47 \mathrm{~B})$ & 109.5 \\
\hline $\mathrm{C}(37)-\mathrm{C}(40)-\mathrm{H}(40 \mathrm{~B})$ & 109.5 & $\mathrm{C}(45)-\mathrm{C}(47)-\mathrm{H}(47 \mathrm{C})$ & 109.5 \\
\hline $\mathrm{H}(40 \mathrm{~A})-\mathrm{C}(40)-\mathrm{H}(40 \mathrm{~B})$ & 109.5 & $\mathrm{H}(47 \mathrm{~A})-\mathrm{C}(47)-\mathrm{H}(47 \mathrm{C})$ & 109.5 \\
\hline $\mathrm{C}(37)-\mathrm{C}(40)-\mathrm{H}(40 \mathrm{C})$ & 109.5 & $\mathrm{H}(47 \mathrm{~B})-\mathrm{C}(47)-\mathrm{H}(47 \mathrm{C})$ & 109.5 \\
\hline $\mathrm{H}(40 \mathrm{~A})-\mathrm{C}(40)-\mathrm{H}(40 \mathrm{C})$ & 109.5 & $\mathrm{C}(45)-\mathrm{C}(48)-\mathrm{H}(48 \mathrm{~A})$ & 109.5 \\
\hline $\mathrm{H}(40 \mathrm{~B})-\mathrm{C}(40)-\mathrm{H}(40 \mathrm{C})$ & 109.5 & $\mathrm{C}(45)-\mathrm{C}(48)-\mathrm{H}(48 \mathrm{~B})$ & 109.5 \\
\hline $\mathrm{C}(43)-\mathrm{C}(41)-\mathrm{C}(44)$ & $109.58(16)$ & $\mathrm{H}(48 \mathrm{~A})-\mathrm{C}(48)-\mathrm{H}(48 \mathrm{~B})$ & 109.5 \\
\hline $\mathrm{C}(43)-\mathrm{C}(41)-\mathrm{C}(42)$ & $108.53(16)$ & $\mathrm{C}(45)-\mathrm{C}(48)-\mathrm{H}(48 \mathrm{C})$ & 109.5 \\
\hline $\mathrm{C}(44)-\mathrm{C}(41)-\mathrm{C}(42)$ & $107.35(16)$ & $\mathrm{H}(48 \mathrm{~A})-\mathrm{C}(48)-\mathrm{H}(48 \mathrm{C})$ & 109.5 \\
\hline $\mathrm{C}(43)-\mathrm{C}(41)-\mathrm{P}(4)$ & $114.92(13)$ & $\mathrm{H}(48 \mathrm{~B})-\mathrm{C}(48)-\mathrm{H}(48 \mathrm{C})$ & 109.5 \\
\hline $\mathrm{C}(44)-\mathrm{C}(41)-\mathrm{P}(4)$ & $108.23(13)$ & $C(50)-C(49)-C(49) \# 1$ & $113.1(3)$ \\
\hline $\mathrm{C}(42)-\mathrm{C}(41)-\mathrm{P}(4)$ & $107.97(13)$ & $\mathrm{C}(50)-\mathrm{C}(49)-\mathrm{H}(49 \mathrm{~A})$ & 109.0 \\
\hline $\mathrm{C}(41)-\mathrm{C}(42)-\mathrm{H}(42 \mathrm{~A})$ & 109.5 & $\mathrm{C}(49) \# 1-\mathrm{C}(49)-\mathrm{H}(49 \mathrm{~A})$ & 109.0 \\
\hline $\mathrm{C}(41)-\mathrm{C}(42)-\mathrm{H}(42 \mathrm{~B})$ & 109.5 & $\mathrm{C}(50)-\mathrm{C}(49)-\mathrm{H}(49 \mathrm{~B})$ & 109.0 \\
\hline $\mathrm{H}(42 \mathrm{~A})-\mathrm{C}(42)-\mathrm{H}(42 \mathrm{~B})$ & 109.5 & $\mathrm{C}(49) \# 1-\mathrm{C}(49)-\mathrm{H}(49 \mathrm{~B})$ & 109.0 \\
\hline $\mathrm{C}(41)-\mathrm{C}(42)-\mathrm{H}(42 \mathrm{C})$ & 109.5 & $\mathrm{H}(49 \mathrm{~A})-\mathrm{C}(49)-\mathrm{H}(49 \mathrm{~B})$ & 107.8 \\
\hline $\mathrm{H}(42 \mathrm{~A})-\mathrm{C}(42)-\mathrm{H}(42 \mathrm{C})$ & 109.5 & $C(49)-C(50)-C(51)$ & $112.6(3)$ \\
\hline $\mathrm{H}(42 \mathrm{~B})-\mathrm{C}(42)-\mathrm{H}(42 \mathrm{C})$ & 109.5 & $\mathrm{C}(49)-\mathrm{C}(50)-\mathrm{H}(50 \mathrm{~A})$ & 109.1 \\
\hline $\mathrm{C}(41)-\mathrm{C}(43)-\mathrm{H}(43 \mathrm{~A})$ & 109.5 & $\mathrm{C}(51)-\mathrm{C}(50)-\mathrm{H}(50 \mathrm{~A})$ & 109.1 \\
\hline $\mathrm{C}(41)-\mathrm{C}(43)-\mathrm{H}(43 \mathrm{~B})$ & 109.5 & $\mathrm{C}(49)-\mathrm{C}(50)-\mathrm{H}(50 \mathrm{~B})$ & 109.1 \\
\hline $\mathrm{H}(43 \mathrm{~A})-\mathrm{C}(43)-\mathrm{H}(43 \mathrm{~B})$ & 109.5 & $\mathrm{C}(51)-\mathrm{C}(50)-\mathrm{H}(50 \mathrm{~B})$ & 109.1 \\
\hline $\mathrm{C}(41)-\mathrm{C}(43)-\mathrm{H}(43 \mathrm{C})$ & 109.5 & $\mathrm{H}(50 \mathrm{~A})-\mathrm{C}(50)-\mathrm{H}(50 \mathrm{~B})$ & 107.8 \\
\hline $\mathrm{H}(43 \mathrm{~A})-\mathrm{C}(43)-\mathrm{H}(43 \mathrm{C})$ & 109.5 & $\mathrm{C}(50)-\mathrm{C}(51)-\mathrm{H}(51 \mathrm{~A})$ & 109.5 \\
\hline $\mathrm{H}(43 \mathrm{~B})-\mathrm{C}(43)-\mathrm{H}(43 \mathrm{C})$ & 109.5 & $\mathrm{C}(50)-\mathrm{C}(51)-\mathrm{H}(51 \mathrm{~B})$ & 109.5 \\
\hline $\mathrm{C}(41)-\mathrm{C}(44)-\mathrm{H}(44 \mathrm{~A})$ & 109.5 & $\mathrm{H}(51 \mathrm{~A})-\mathrm{C}(51)-\mathrm{H}(51 \mathrm{~B})$ & 109.5 \\
\hline $\mathrm{C}(41)-\mathrm{C}(44)-\mathrm{H}(44 \mathrm{~B})$ & 109.5 & $\mathrm{C}(50)-\mathrm{C}(51)-\mathrm{H}(51 \mathrm{C})$ & 109.5 \\
\hline $\mathrm{H}(44 \mathrm{~A})-\mathrm{C}(44)-\mathrm{H}(44 \mathrm{~B})$ & 109.5 & $\mathrm{H}(51 \mathrm{~A})-\mathrm{C}(51)-\mathrm{H}(51 \mathrm{C})$ & 109.5 \\
\hline $\mathrm{C}(41)-\mathrm{C}(44)-\mathrm{H}(44 \mathrm{C})$ & 109.5 & $\mathrm{H}(51 \mathrm{~B})-\mathrm{C}(51)-\mathrm{H}(51 \mathrm{C})$ & 109.5 \\
\hline
\end{tabular}

Symmetry transformations used to generate equivalent atoms: $\# 1-\mathrm{x}+2,-\mathrm{y},-\mathrm{z}+2$ 
Table S-8. Torsion angles $\left[{ }^{\circ}\right]$ for complex 2 .

\begin{tabular}{|c|c|c|c|}
\hline $\mathrm{C}(1)-\operatorname{Ir}(1)-\mathrm{N}(1)-\mathrm{N}(2)$ & $-12(12)$ & $\operatorname{Ir}(1)-\mathrm{C}(1)-\mathrm{C}(6)-\mathrm{C}(8)$ & $-0.5(2)$ \\
\hline $\mathrm{P}(1)-\operatorname{Ir}(1)-\mathrm{N}(1)-\mathrm{N}(2)$ & $-147(8)$ & $\mathrm{C}(3)-\mathrm{C}(2)-\mathrm{C}(7)-\mathrm{P}(1)$ & $-157.69(16)$ \\
\hline $\mathrm{P}(2)-\operatorname{Ir}(1)-\mathrm{N}(1)-\mathrm{N}(2)$ & $32(8)$ & $\mathrm{C}(1)-\mathrm{C}(2)-\mathrm{C}(7)-\mathrm{P}(1)$ & $21.2(2)$ \\
\hline $\operatorname{Ir}(1)-\mathrm{N}(1)-\mathrm{N}(2)-\operatorname{Ir}(2)$ & $-74(30)$ & $\mathrm{C}(13)-\mathrm{P}(1)-\mathrm{C}(7)-\mathrm{C}(2)$ & $-155.14(13)$ \\
\hline $\mathrm{C}(25)-\operatorname{Ir}(2)-\mathrm{N}(2)-\mathrm{N}(1)$ & $-53(29)$ & $\mathrm{C}(9)-\mathrm{P}(1)-\mathrm{C}(7)-\mathrm{C}(2)$ & $89.67(14)$ \\
\hline $\mathrm{P}(3)-\operatorname{Ir}(2)-\mathrm{N}(2)-\mathrm{N}(1)$ & $-47(26)$ & $\operatorname{Ir}(1)-P(1)-C(7)-C(2)$ & $-30.21(14)$ \\
\hline $\mathrm{P}(4)-\operatorname{Ir}(2)-\mathrm{N}(2)-\mathrm{N}(1)$ & $133(26)$ & $\mathrm{C}(5)-\mathrm{C}(6)-\mathrm{C}(8)-\mathrm{P}(2)$ & $-156.83(15)$ \\
\hline $\mathrm{N}(1)-\operatorname{Ir}(1)-\mathrm{P}(1)-\mathrm{C}(7)$ & $-156.45(8)$ & $\mathrm{C}(1)-\mathrm{C}(6)-\mathrm{C}(8)-\mathrm{P}(2)$ & $22.8(2)$ \\
\hline $\mathrm{C}(1)-\operatorname{Ir}(1)-\mathrm{P}(1)-\mathrm{C}(7)$ & $24.06(8)$ & $\mathrm{C}(17)-\mathrm{P}(2)-\mathrm{C}(8)-\mathrm{C}(6)$ & $-154.61(13)$ \\
\hline $\mathrm{P}(2)-\operatorname{Ir}(1)-\mathrm{P}(1)-\mathrm{C}(7)$ & $26.31(9)$ & $\mathrm{C}(21)-\mathrm{P}(2)-\mathrm{C}(8)-\mathrm{C}(6)$ & 89.72(14) \\
\hline $\mathrm{N}(1)-\operatorname{Ir}(1)-\mathrm{P}(1)-\mathrm{C}(13)$ & $-43.61(9)$ & $\operatorname{Ir}(1)-\mathrm{P}(2)-\mathrm{C}(8)-\mathrm{C}(6)$ & $-31.11(13)$ \\
\hline $\mathrm{C}(1)-\operatorname{Ir}(1)-\mathrm{P}(1)-\mathrm{C}(13)$ & $136.90(9)$ & $\mathrm{C}(7)-\mathrm{P}(1)-\mathrm{C}(9)-\mathrm{C}(12)$ & $-167.04(13)$ \\
\hline $\mathrm{P}(2)-\operatorname{Ir}(1)-\mathrm{P}(1)-\mathrm{C}(13)$ & $139.15(8)$ & $\mathrm{C}(13)-\mathrm{P}(1)-\mathrm{C}(9)-\mathrm{C}(12)$ & $83.71(15)$ \\
\hline N(1)-Ir(1)-P(1)-C(9) & $91.67(8)$ & $\operatorname{Ir}(1)-\mathrm{P}(1)-\mathrm{C}(9)-\mathrm{C}(12)$ & $-55.51(14)$ \\
\hline $\mathrm{C}(1)-\operatorname{Ir}(1)-\mathrm{P}(1)-\mathrm{C}(9)$ & $-87.82(8)$ & $\mathrm{C}(7)-\mathrm{P}(1)-\mathrm{C}(9)-\mathrm{C}(10)$ & $69.83(16)$ \\
\hline $\mathrm{P}(2)-\operatorname{Ir}(1)-\mathrm{P}(1)-\mathrm{C}(9)$ & $-85.57(8)$ & $\mathrm{C}(13)-\mathrm{P}(1)-\mathrm{C}(9)-\mathrm{C}(10)$ & $-39.41(17)$ \\
\hline $\mathrm{N}(1)-\operatorname{Ir}(1)-\mathrm{P}(2)-\mathrm{C}(8)$ & $-155.29(8)$ & $\operatorname{Ir}(1)-\mathrm{P}(1)-\mathrm{C}(9)-\mathrm{C}(10)$ & $-178.63(12)$ \\
\hline $\mathrm{C}(1)-\operatorname{Ir}(1)-\mathrm{P}(2)-\mathrm{C}(8)$ & $24.21(8)$ & $\mathrm{C}(7)-\mathrm{P}(1)-\mathrm{C}(9)-\mathrm{C}(11)$ & $-51.01(15)$ \\
\hline $\mathrm{P}(1)-\operatorname{Ir}(1)-\mathrm{P}(2)-\mathrm{C}(8)$ & $21.96(9)$ & $\mathrm{C}(13)-\mathrm{P}(1)-\mathrm{C}(9)-\mathrm{C}(11)$ & $-160.25(13)$ \\
\hline $\mathrm{N}(1)-\operatorname{Ir}(1)-\mathrm{P}(2)-\mathrm{C}(17)$ & $-44.01(8)$ & $\operatorname{Ir}(1)-\mathrm{P}(1)-\mathrm{C}(9)-\mathrm{C}(11)$ & $60.53(14)$ \\
\hline $\mathrm{C}(1)-\operatorname{Ir}(1)-\mathrm{P}(2)-\mathrm{C}(17)$ & $135.49(9)$ & $\mathrm{C}(7)-\mathrm{P}(1)-\mathrm{C}(13)-\mathrm{C}(16)$ & $-166.24(14)$ \\
\hline $\mathrm{P}(1)-\operatorname{Ir}(1)-\mathrm{P}(2)-\mathrm{C}(17)$ & $133.25(8)$ & $\mathrm{C}(9)-\mathrm{P}(1)-\mathrm{C}(13)-\mathrm{C}(16)$ & $-56.28(15)$ \\
\hline $\mathrm{N}(1)-\operatorname{Ir}(1)-\mathrm{P}(2)-\mathrm{C}(21)$ & $92.80(8)$ & $\operatorname{Ir}(1)-\mathrm{P}(1)-\mathrm{C}(13)-\mathrm{C}(16)$ & $80.64(14)$ \\
\hline$C(1)-\operatorname{Ir}(1)-P(2)-C(21)$ & $-87.70(8)$ & $\mathrm{C}(7)-\mathrm{P}(1)-\mathrm{C}(13)-\mathrm{C}(15)$ & $76.78(14)$ \\
\hline $\mathrm{P}(1)-\operatorname{Ir}(1)-\mathrm{P}(2)-\mathrm{C}(21)$ & $-89.94(8)$ & $\mathrm{C}(9)-\mathrm{P}(1)-\mathrm{C}(13)-\mathrm{C}(15)$ & $-173.27(13)$ \\
\hline $\mathrm{N}(2)-\operatorname{Ir}(2)-\mathrm{P}(3)-\mathrm{C}(31)$ & $155.62(7)$ & $\operatorname{Ir}(1)-\mathrm{P}(1)-\mathrm{C}(13)-\mathrm{C}(15)$ & $-36.34(15)$ \\
\hline$C(25)-\operatorname{Ir}(2)-P(3)-C(31)$ & $-24.42(8)$ & $\mathrm{C}(7)-\mathrm{P}(1)-\mathrm{C}(13)-\mathrm{C}(14)$ & $-41.21(17)$ \\
\hline $\mathrm{P}(4)-\operatorname{Ir}(2)-\mathrm{P}(3)-\mathrm{C}(31)$ & $-27.04(8)$ & $\mathrm{C}(9)-\mathrm{P}(1)-\mathrm{C}(13)-\mathrm{C}(14)$ & $68.74(17)$ \\
\hline $\mathrm{N}(2)-\operatorname{Ir}(2)-\mathrm{P}(3)-\mathrm{C}(33)$ & $43.44(8)$ & $\operatorname{Ir}(1)-\mathrm{P}(1)-\mathrm{C}(13)-\mathrm{C}(14)$ & $-154.33(13)$ \\
\hline$C(25)-\operatorname{Ir}(2)-P(3)-C(33)$ & $-136.60(9)$ & $\mathrm{C}(8)-\mathrm{P}(2)-\mathrm{C}(17)-\mathrm{C}(20)$ & $-163.95(13)$ \\
\hline $\mathrm{P}(4)-\operatorname{Ir}(2)-\mathrm{P}(3)-\mathrm{C}(33)$ & $-139.22(8)$ & $\mathrm{C}(21)-\mathrm{P}(2)-\mathrm{C}(17)-\mathrm{C}(20)$ & $-54.20(15)$ \\
\hline $\mathrm{N}(2)-\operatorname{Ir}(2)-\mathrm{P}(3)-\mathrm{C}(37)$ & $-92.84(8)$ & $\operatorname{Ir}(1)-P(2)-C(17)-C(20)$ & $84.39(13)$ \\
\hline$C(25)-\operatorname{Ir}(2)-P(3)-C(37)$ & $87.12(9)$ & $\mathrm{C}(8)-\mathrm{P}(2)-\mathrm{C}(17)-\mathrm{C}(18)$ & $78.81(13)$ \\
\hline$P(4)-\operatorname{Ir}(2)-P(3)-C(37)$ & $84.50(8)$ & $\mathrm{C}(21)-\mathrm{P}(2)-\mathrm{C}(17)-\mathrm{C}(18)$ & $-171.44(11)$ \\
\hline $\mathrm{N}(2)-\operatorname{Ir}(2)-\mathrm{P}(4)-\mathrm{C}(32)$ & $155.86(8)$ & $\operatorname{Ir}(1)-P(2)-C(17)-C(18)$ & $-32.85(14)$ \\
\hline$C(25)-\operatorname{Ir}(2)-P(4)-C(32)$ & $-24.10(8)$ & $\mathrm{C}(8)-\mathrm{P}(2)-\mathrm{C}(17)-\mathrm{C}(19)$ & $-39.39(16)$ \\
\hline$P(3)-\operatorname{Ir}(2)-P(4)-C(32)$ & $-21.47(9)$ & $\mathrm{C}(21)-\mathrm{P}(2)-\mathrm{C}(17)-\mathrm{C}(19)$ & $70.36(15)$ \\
\hline $\mathrm{N}(2)-\operatorname{Ir}(2)-\mathrm{P}(4)-\mathrm{C}(45)$ & $43.97(8)$ & $\operatorname{Ir}(1)-P(2)-C(17)-C(19)$ & $-151.05(12)$ \\
\hline$C(25)-\operatorname{Ir}(2)-P(4)-C(45)$ & $-135.98(8)$ & $\mathrm{C}(8)-\mathrm{P}(2)-\mathrm{C}(21)-\mathrm{C}(24)$ & $-166.88(14)$ \\
\hline $\mathrm{P}(3)-\operatorname{Ir}(2)-\mathrm{P}(4)-\mathrm{C}(45)$ & $-133.36(8)$ & $\mathrm{C}(17)-\mathrm{P}(2)-\mathrm{C}(21)-\mathrm{C}(24)$ & $84.41(15)$ \\
\hline $\mathrm{N}(2)-\operatorname{Ir}(2)-\mathrm{P}(4)-\mathrm{C}(41)$ & $-92.42(8)$ & $\operatorname{Ir}(1)-P(2)-C(21)-C(24)$ & $-55.53(15)$ \\
\hline$C(25)-\operatorname{Ir}(2)-P(4)-C(41)$ & $87.63(8)$ & $\mathrm{C}(8)-\mathrm{P}(2)-\mathrm{C}(21)-\mathrm{C}(23)$ & $69.68(15)$ \\
\hline $\mathrm{P}(3)-\operatorname{Ir}(2)-\mathrm{P}(4)-\mathrm{C}(41)$ & $90.25(8)$ & $\mathrm{C}(17)-\mathrm{P}(2)-\mathrm{C}(21)-\mathrm{C}(23)$ & $-39.03(16)$ \\
\hline $\mathrm{N}(1)-\operatorname{Ir}(1)-\mathrm{C}(1)-\mathrm{C}(2)$ & $-153(5)$ & $\operatorname{Ir}(1)-P(2)-C(21)-C(23)$ & $-178.97(12)$ \\
\hline $\mathrm{P}(1)-\operatorname{Ir}(1)-\mathrm{C}(1)-\mathrm{C}(2)$ & $-17.47(14)$ & $\mathrm{C}(8)-\mathrm{P}(2)-\mathrm{C}(21)-\mathrm{C}(22)$ & $-51.11(15)$ \\
\hline $\mathrm{P}(2)-\operatorname{Ir}(1)-\mathrm{C}(1)-\mathrm{C}(2)$ & $163.28(16)$ & $\mathrm{C}(17)-\mathrm{P}(2)-\mathrm{C}(21)-\mathrm{C}(22)$ & $-159.82(13)$ \\
\hline $\mathrm{N}(1)-\operatorname{Ir}(1)-\mathrm{C}(1)-\mathrm{C}(6)$ & $27(5)$ & $\operatorname{Ir}(1)-P(2)-C(21)-C(22)$ & $60.24(14)$ \\
\hline$P(1)-\operatorname{Ir}(1)-C(1)-C(6)$ & $162.48(16)$ & $\mathrm{N}(2)-\operatorname{Ir}(2)-\mathrm{C}(25)-\mathrm{C}(30)$ & $-157(9)$ \\
\hline $\mathrm{P}(2)-\operatorname{Ir}(1)-\mathrm{C}(1)-\mathrm{C}(6)$ & $-16.77(14)$ & $P(3)-\operatorname{Ir}(2)-C(25)-C(30)$ & $-162.31(15)$ \\
\hline $\mathrm{C}(6)-\mathrm{C}(1)-\mathrm{C}(2)-\mathrm{C}(3)$ & $-0.1(3)$ & $P(4)-\operatorname{Ir}(2)-C(25)-C(30)$ & $16.81(14)$ \\
\hline $\operatorname{Ir}(1)-\mathrm{C}(1)-\mathrm{C}(2)-\mathrm{C}(3)$ & $179.88(15)$ & $\mathrm{N}(2)-\operatorname{Ir}(2)-\mathrm{C}(25)-\mathrm{C}(26)$ & $22(10)$ \\
\hline$C(6)-C(1)-C(2)-C(7)$ & $-178.96(17)$ & $P(3)-\operatorname{Ir}(2)-C(25)-C(26)$ & $17.07(14)$ \\
\hline $\operatorname{Ir}(1)-C(1)-C(2)-C(7)$ & $1.0(2)$ & $\mathrm{P}(4)-\operatorname{Ir}(2)-\mathrm{C}(25)-\mathrm{C}(26)$ & $-163.81(15)$ \\
\hline $\mathrm{C}(1)-\mathrm{C}(2)-\mathrm{C}(3)-\mathrm{C}(4)$ & $1.0(3)$ & $C(30)-C(25)-C(26)-C(27)$ & $0.6(3)$ \\
\hline $\mathrm{C}(7)-\mathrm{C}(2)-\mathrm{C}(3)-\mathrm{C}(4)$ & $179.90(19)$ & $\operatorname{Ir}(2)-C(25)-C(26)-C(27)$ & $-178.85(14)$ \\
\hline$C(2)-C(3)-C(4)-C(5)$ & $-1.0(3)$ & $C(30)-C(25)-C(26)-C(31)$ & 179.61(16) \\
\hline$C(3)-C(4)-C(5)-C(6)$ & $0.0(3)$ & $\operatorname{Ir}(2)-C(25)-C(26)-C(31)$ & $0.2(2)$ \\
\hline$C(4)-C(5)-C(6)-C(1)$ & $1.0(3)$ & $\mathrm{C}(25)-\mathrm{C}(26)-\mathrm{C}(27)-\mathrm{C}(28)$ & $-1.3(3)$ \\
\hline$C(4)-C(5)-C(6)-C(8)$ & $-179.34(18)$ & $\mathrm{C}(31)-\mathrm{C}(26)-\mathrm{C}(27)-\mathrm{C}(28)$ & $179.64(17)$ \\
\hline $\mathrm{C}(2)-\mathrm{C}(1)-\mathrm{C}(6)-\mathrm{C}(5)$ & $-1.0(3)$ & $\mathrm{C}(26)-\mathrm{C}(27)-\mathrm{C}(28)-\mathrm{C}(29)$ & $0.3(3)$ \\
\hline $\operatorname{Ir}(1)-C(1)-C(6)-C(5)$ & $179.10(14)$ & $\mathrm{C}(27)-\mathrm{C}(28)-\mathrm{C}(29)-\mathrm{C}(30)$ & $1.4(3)$ \\
\hline $\mathrm{C}(2)-\mathrm{C}(1)-\mathrm{C}(6)-\mathrm{C}(8)$ & $179.41(17)$ & $\mathrm{C}(28)-\mathrm{C}(29)-\mathrm{C}(30)-\mathrm{C}(25)$ & $-2.2(3)$ \\
\hline
\end{tabular}




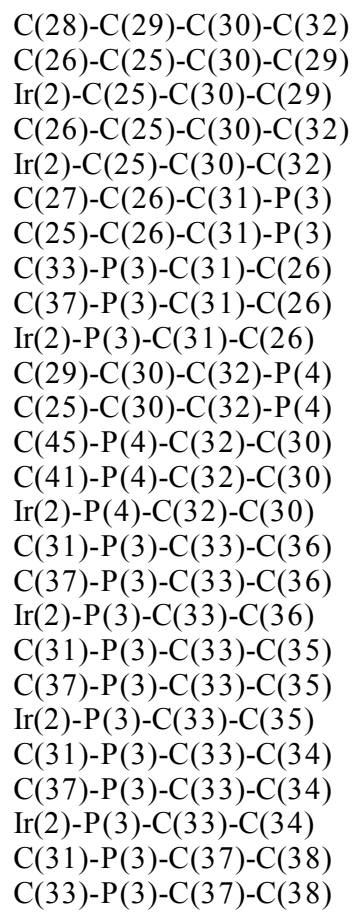

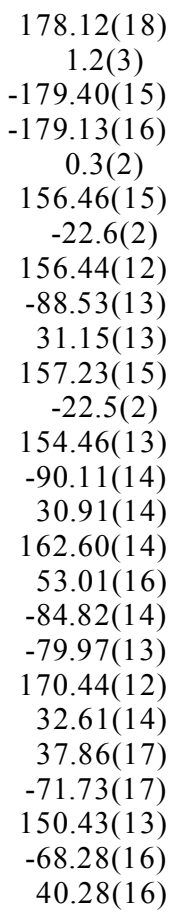

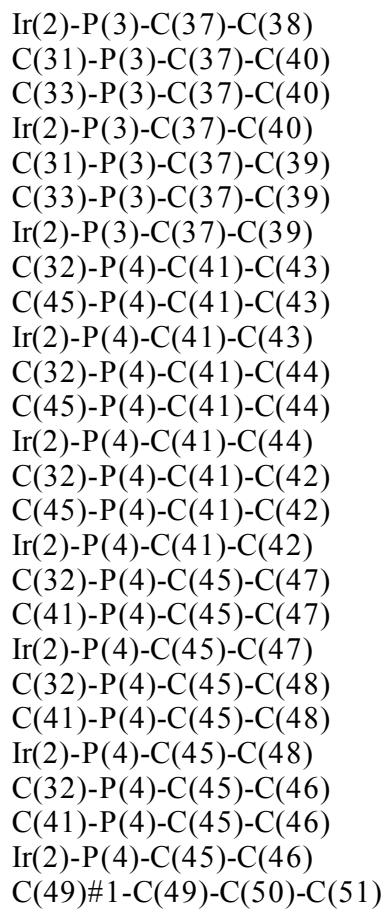

Symmetry transformations used to generate equivalent atoms: $\# 1-\mathrm{x}+2,-\mathrm{y},-\mathrm{z}+2$ 